ACCEPTED MANUSCRIPT

\title{
Performance evaluation of a novel multi-pinhole collimator for dopamine transporter SPECT
}

To cite this article before publication: Kristian Tecklenburg et al 2020 Phys. Med. Biol. in press https://doi.org/10.1088/1361-6560/ab9067

\section{Manuscript version: Accepted Manuscript}

Accepted Manuscript is "the version of the article accepted for publication including all changes made as a result of the peer review process, and which may also include the addition to the article by IOP Publishing of a header, an article ID, a cover sheet and/or an 'Accepted

Manuscript' watermark, but excluding any other editing, typesetting or other changes made by IOP Publishing and/or its licensors"

This Accepted Manuscript is @ 2020 Institute of Physics and Engineering in Medicine.

During the embargo period (the 12 month period from the publication of the Version of Record of this article), the Accepted Manuscript is fully protected by copyright and cannot be reused or reposted elsewhere.

As the Version of Record of this article is going to be / has been published on a subscription basis, this Accepted Manuscript is available for reuse under a CC BY-NC-ND 3.0 licence after the 12 month embargo period.

After the embargo period, everyone is permitted to use copy and redistribute this article for non-commercial purposes only, provided that they adhere to all the terms of the licence https://creativecommons.org/licences/by-nc-nd/3.0

Although reasonable endeavours have been taken to obtain all necessary permissions from third parties to include their copyrighted content within this article, their full citation and copyright line may not be present in this Accepted Manuscript version. Before using any content from this article, please refer to the Version of Record on IOPscience once published for full citation and copyright details, as permissions will likely be required. All third party content is fully copyright protected, unless specifically stated otherwise in the figure caption in the Version of Record.

View the article online for updates and enhancements. 


\section{Performance evaluation of a novel multi-pinhole collimator for dopamine}

\section{transporter SPECT}

K. Tecklenburg ${ }^{1,2}$, A. Forgács ${ }^{3}$, I. Apostolova ${ }^{1}$, W. Lehnert ${ }^{1}$, S. Klutmann ${ }^{1}$,J. Csirik ${ }^{4}$, E. Garutti ${ }^{2}$, R.

\section{Buchert $^{1}$}

${ }^{1}$ Department of Diagnostic and Interventional Radiology and Nuclear Medicine, University Medical Center Hamburg-Eppendorf, Hamburg, Germany

${ }^{2}$ Institute of Experimental Physics, Faculty of Mathematics, Informatics and Natural Sciences, University of Hamburg, Hamburg, Germany

${ }^{3}$ Scanomed Nuclear Medicine Center Debrecen, Debrecen, Hungary

${ }^{4}$ Department of Computer Algorithms and Artificial Intelligence, University of Szeged, Szeged, Hungary

Corresponding author: Ralph Buchert, Department of Diagnostic and Interventional Radiology and Nuclear Medicine, University Medical Center Hamburg-Eppendorf, Martinistr. 52, 20246 Hamburg, Germany, Email: r.buchert@uke.de, Phone: +49 (0)40 7410-54347, Fax: +49 (0)40 7410-40265 


\begin{abstract}
Purpose: There is a tradeoff between spatial resolution and count sensitivity in SPECT with conventional collimators. Multi-pinhole (MPH) collimator technology has potential for concurrent improvement of resolution and sensitivity in clinical SPECT of 'small' organs. This study evaluated a novel MPH collimator specifically designed for dopamine transporter (DAT) SPECT with a triple-head SPECT camera.
\end{abstract}

Methods: Count sensitivity was measured with a ${ }^{99 \mathrm{~m}} \mathrm{Tc}$ point source placed on the lattice points of a $1 \mathrm{~cm}$ grid covering the whole field-of-view (FOV). Spatial resolution was assessed with a Derenzo type hot rod phantom. An anthropomorphic striatum phantom was scanned with total activity representative of a typical patient scan and different striatum-to-background activity concentration ratios. Recovery of striatum-tobackground contrast was assessed by the contrast-recovery-coefficient. Measurements were repeated with double-head SPECT with fan-beam or low-energy-high-resolution-high-sensitivity (LEHRHS) collimators. A patient referred to DAT SPECT because of suspicion of Parkinson's disease was scanned with both LEHRHS and MPH collimators after a single tracer injection.

Results: The axial MPH sensitivity profile was almost symmetrical around its peak, although it was shifted $7 \mathrm{~cm}$ towards the patient to simplify positioning. Peak sensitivity of the triple-head MPH system in the center of the FOV was $620 \mathrm{cps} / \mathrm{MBq}$ compared to $225 \mathrm{cps} / \mathrm{MBq}$ for the double-head fan-beam system. Sensitivity of the MPH system decreased towards the edges of the FOV. The full width of the sensitivity profile at $200 \mathrm{cps} / \mathrm{MBq}$ was $21 \mathrm{~cm}$ transaxially and $11 \mathrm{~cm}$ axially. In MPH SPECT of the Derenzo phantom all rods with $\geq 5 \mathrm{~mm}$ diameter were clearly visible. MPH SPECT improved striatal contrast recovery by $\geq$ $20 \%$ compared to fan-beam SPECT. The patient scan demonstrated good image quality of MPH SPECT with almost PET-like delineation of putamen and caudate nucleus.

Conclusions: SPECT with dedicated MPH collimators provides considerable improvement of the resolution-sensitivity tradeoff in DAT SPECT compared to SPECT with fan-beam or LEHRHS collimators.

Key words: dopamine transporter; SPECT; collimator; pinhole; performance; sensitivity; spatial resolution 


\section{Introduction}

Parkinson's disease (PD) is the second most common neurodegenerative disorder after Alzheimer's disease (Twelves et al., 2003). PD is characterized by bradykinesia, resting tremor, rigidity, and postural instability (Gibb and Lees, 1988). This combination of symptoms, referred to as parkinsonian syndrome (PS), can also be caused by cerebrovascular disease, drugs, head trauma, inflammatory or metabolic disorder, and then is denoted as 'secondary' PS. The differentiation between PD and secondary PS is highly relevant, because secondary PS might be treated more effectively than PD, some secondary PS may be fully cured. Yet, the clinical, that is, symptom-based differentiation between PD and secondary PS is challenging in a considerable fraction of patients, particularly at early disease stages with mild symptoms and in patients with atypical presentation, often subsumed under the term "clinical uncertain parkinsonian syndrome" (CUPS) (Buchert et al., 2019).

Clinical practice guidelines recommend single photon emission computed tomography (SPECT) with the dopamine transporter (DAT) ligand ${ }^{123}$ I-FP-CIT to support differentiation between PD and secondary PS in CUPS (Berardelli et al., 2013; Darcourt et al., 2010; Djang et al., 2012). The rationale is detection of PD-characteristic loss of dopaminergic neurons in the brain (nigrostriatal degeneration) by DAT SPECT (2017; Berardelli et al., 2013). Secondary PS are not associated with nigrostriatal degeneration and therefore present with normal findings in DAT SPECT.

${ }^{123}$ I-FP-CIT has good pharmacokinetic properties (Andringa et al., 2005; Geisler et al., 2015). The major limitation of DAT SPECT is limited image quality provided by conventional SPECT technology with respect to spatial resolution, statistical noise and quantitative accuracy and precision. As a consequence, DAT SPECT allows reliable detection of nigrostriatal degeneration especially in advanced stages of PD, when the loss of DAT in the more severely affected putamen exceeds $50 \%$ and patients develop movement problems (Bernheimer et al., 1973; Fearnley and Lees, 1991; Niznik et al., 1991). Yet, there are early signs of PD such as smell loss and rapid eye movement sleep and behavior disorder that can precede motor symptoms by several years, but are not particularly specific for PD. As soon as disease-modifying drugs for treatment of PD will be available, it will be important to detect PD also in these early stages, when the loss of putaminal DAT is considerably smaller than $50 \%$, because the chance to moderate the course of PD by these drugs will be better at early disease stages (Kim, 2017). DAT SPECT is a promising diagnostic 
modality for early detection of PD (before the occurrence of characteristic motor symptoms), but best possible image quality is required for this purpose (Chahine et al., 2019; Iranzo et al., 2017; Meles et al., 2017).

The limited image quality of conventional SPECT technology is associated with the tradeoff between spatial resolution and statistical noise due to the use of parallel-hole collimators to define the line of response of photons detected by the scintillation detector for image reconstruction (Van Audenhaege et al., 2015a). In brain SPECT, the resolution-noise tradeoff can be considerably improved by the use/of fanbeam collimators that provide $20-40 \%$ increase of count sensitivity at about the same spatial resolution compared to parallel-hole SPECT (Van Audenhaege et al., 2015a; Akiyama and Yui, 1991).

Yet, multi-pinhole (MPH) technology has considerably larger potential for concurrent improvement of spatial resolution and count sensitivity in brain SPECT than fan-beam technology (Van Audenhaege et al., 2015a; Azazrm et al., 2015; Beekman and Van Der Have, 2007; Beekman et al., 2005; Ivashchenko et al., 2014; Vaissier et al., 2012; Vastenhouw et al., 2007). The basic idea of MPH technology is to focus on small objects (improved count sensitivity) such that the image of the object on the scintillation crystal is considerably zoomed (improved spatial resolution by reduced impact of the intrinsic spatial resolution of the scintillation detector). This explains that MPH technology has been successful in small animal SPECT (Lange et al., 2014a; Apostolova et al., 2015; Apostolova et al., 2012; Alvarez-Fischer et al., 2007; Andringa et al., 2005; Bal et al., 2008; Difilippo, 2008; Nuyts et al., 2009; Peterson and Shokouhi, 2012; Scherfler et al., 2002; Shokouhi et al., 2009; Vanhove et al., 2008). Large detectors equipped with specifically designed MPH collimators allow up to quarter-millimeter-resolution molecular mouse SPECT (Ivashchenko et al., 2014).

Transfer of MPH technology to SPECT of 'small' organs in humans including heart (Si et al., 2016; Yan et al., 2016), thyroid (Bae et al., 2017), and brain (Chen et al., 2017; Salvado et al., 2015; Chen et al., 2018; Lee et al., 2014; Spilker et al., 2008) is not straightforward, because the proportion between the object to be imaged (e.g., human brain) and the size of the scintillation detector is less favorable in patients than in mice (less zooming possible). Thus, human MPH SPECT requires careful optimization of MPH design with respect to the number of pinholes per detector, spatial arrangement of the pinholes relative to each other, pinhole shape and orientation for the specific application (Cao et al., 2005; Johnson et al., 2016; Nillius and Danielsson, 2010; Rentmeester et al., 2007; Vunckx et al., 2009). 
The aim of the present study was to evaluate the performance of a novel MPH collimator specifically designed for clinical DAT SPECT with a triple-head SPECT (or SPECT/CT) system. 


\title{
Materials and methods
}

\author{
Triple-head SPECT system
}

The AnyScan ${ }^{\circledR}$ Trio (Mediso Medical Imaging Systems, Budapest, Hungary) is a general purpose triplehead SPECT system. Each head is equipped with a 3/8" NaI(Tl) detector of $585 \mathrm{~mm}$ (transaxial) x $470 \mathrm{~mm}$ (axial), $16 \mathrm{~mm}$ lightguide, and 94 photomultipliers. MPH collimators specifically designed for DAT SPECT are available for this system in addition to conventional parallel-hole collimators.

\section{Multi-pinhole collimator}

The MPH collimator (Fig. 1) was designed for targeted SPECT imaging with high count sensitivity at the striatum and minimum multiplexing artefacts by combination of overlapping and non-overlapping projections (Van Audenhaege et al., 2015b; Lin, 2013). The collimator features a solid tungsten aperture plate of $18 \mathrm{~mm}$ thickness with 20 pinholes approximately arranged in 5 axially oriented columns and 4 transaxially oriented rows (Fig. 1). The MPH was designed for a total SPECT field-of-view (FOV) of 220 mm transaxial diameter and $180 \mathrm{~mm}$ axial length (Fig. $1 \mathrm{~A})$.

The pinholes of the central axial column, called first order pinholes (Figure $1 \mathrm{C}$ ), cover the central FOV (CFOV), a cylinder of $120 \mathrm{~mm}$ transaxial diameter and $120 \mathrm{~mm}$ axial length that is centered in the total FOV in both axial and transaxial direction. The second order pinholes in the two axial columns neighboring the central column also cover the CFOV. The two inner pinholes in each of the two $2^{\text {nd }}$ order pinhole rows are slightly shifted towards the center of the field-of-view in both axial and transaxial direction (Fig. 1C). Similarly, the two inner pinholes of the $1^{\text {st }}$ order pinhole row are slightly shifted towards the center of the field-of-view in axial direction. The third order pinholes of the two outer axial columns cover the rest of the total FOV together with a small part of the CFOV. Multiplexing can occur only by transaxial overlap between the projections of second and third order pinholes (Fig. 1 D, E). Projections of first order pinholes are not affected by multiplexing, independent of the object to be imaged, projections from $2^{\text {nd }}$ and $3^{\text {rd }}$ order pinholes are not affected by multiplexing with neighboring pinholes of the same order.

Each pinhole is shaped like a double truncated pyramid with one rectangular pyramid base facing the patient, the other rectangular pyramid base facing the detector (Fig. 1A). The center of the double truncated 
pyramid (defining the focal plane) is located $5 \mathrm{~mm}$ from the outer surface of the aperture plate (facing the patient) and $13 \mathrm{~mm}$ from its inner surface (facing the detector). The focus of the pinhole is a square of about $4 \mathrm{~mm}$ edge length for first and second order pinholes. It is a square of about $5 \mathrm{~mm}$ edge length for third order pinholes.

Each double truncated pinhole pyramid is sheared such that its central axis is not orthogonal to the MPH aperture plate but tilted towards the patient. Therefore, the FOV of the MPH collimator is not centered between the MPH aperture plates, but it is shifted by $7 \mathrm{~cm}$ towards the patient (Fig. 1A).

The MPH aperture plate is mounted on a lead blind. The inferior part of the lead blind (facing the patient) is $32 \mathrm{~mm}$ thick in order to efficiently shield photons originating from other parts of the body than the head. All other parts of the lead blind are $12 \mathrm{~mm}$ thick to limit the weight. The lead blind defines the orthogonal distance between the pinhole focal plane and the detector surface to $145 \mathrm{~mm}$. For DAT-SPECT, the rotation radius should be such that the distance between the center-of-rotation (COR) axis and the face of the detector is $285 \mathrm{~mm}$ (the same for all patients). This results in a distance of $140 \mathrm{~mm}$ between the COR axis and the pinhole focal plane. Projection views are acquired in a 256 x 256 matrix with $2.13 \mathrm{~mm}$ x $2.13 \mathrm{~mm}$ pixel size.

The total weight of one MPH collimator (aperture plate and blind) is about $100 \mathrm{~kg}$. The three MPH collimators can be mounted on collimator carts, one cart for two MPH collimators, another cart for the remaining MPH collimator. The same type of carts is used for conventional collimators.

The MPH reconstruction is built upon the iterative one-step-late maximum-a-posteriori expectationmaximization scheme (Green, 1990). A Monte Carlo photon simulation engine is used for forward and backward projection (Buvat and Castiglion, 2002). The forward projector is divided into two parts. The first part is a "direct" projection considering photons directed towards the pinholes and accounting only for attenuation (no scatter) in the patient (based on segmented CT if available). The second part simulates only scatter in the patient, starting from the count density at the current step of the iterative reconstruction. The photons simulated for scatter are emitted isotropically, that is, uniformly in the full $4 \pi$ solid angle. The Woodcock algorithm is used to track the photon path and to simulate Compton scattering (Woodcock et al., 1965). Attenuation of scattered photons is also taken into account. The aperture plate of the MPH collimator and the detector crystal are simulated similarly in both parts, that is, Compton scatter and photo- 
electric effect are considered in both. Variance reduction methods are used such that Compton scatter is limited by the detectable energy defined by the energy window of the acquisition. Only photons with a low weight are removed (bias is restored using Russian roulette), and photons are forced to be detected in the crystal. The intrinsic spatial resolution of the detector is modeled by Gaussian convolution based on calibration data measured during installation of the SPECT system. The backprojection in MPH image reconstruction is a geometric one (with Monte Carlo sampling), it does not match with the forward projection.

Regularization is achieved by filtering between iterations and the beta parameter of the total variation prior used in the maximum-a-posteriori scheme (Panin et al., 1999). Filtering between iterations comprised two independent procedures, bright-dot removal and 3-dimensional bilateral filtering. Bright-dot removal works as follows. For each voxel, the bright-dot filter compares the count density in the considered voxel with the count density in the 26 neighboring voxels in the $3 \times 3 \times 3$ voxels cube centered at the considered voxel. If the current count density in the considered voxel is identified as an outlier, it is replaced by the median count density in the 26 neighboring voxels. The voxel is considered an outlier if the count density in 23 or more of the 26 neighboring voxels differs more than $10 \%$ form the count density in the considered voxel. Bright-dot removal stabilizes the iterative reconstruction particularly in cases with high statistical noise. Bilateral-filtering is a filtering technique for edge-preserving denoising of images including lowcount SPECT (Nakabayashi et al., 2018). In contrast to conventional filtering, the weights of bilateral filters depend not only on the distance of voxels but also on radiometric features such as count density. The latter allows to better preserve edges.

The quality of the Monte Carlo simulation, associated with the number of simulated photons, can be chosen as low (8 million simulated photons), medium (16 million), or high (32 million).

Post-reconstruction filtering was not used in this study.

Order zero attenuation correction was performed after image reconstruction using the post reconstruction attenuation correction method proposed by Chang (Chang, 1978). More precisely, assuming an uniform linear (broad-beam) attenuation coefficient $\mu=0.12 / \mathrm{cm}$, the count density in a given voxel in the reconstructed image was multiplied by the factor $n / \sum_{i=1}^{n} \exp \left(-\mu L_{i}\right)$, where $\mathrm{n}$ is the total number of simulated parallel-hole projections through the voxel in $360^{\circ}$ acquisition, and $L_{i}$ is the distance of the voxel 
to the outer contour of the head. The latter was delineated manually by placing 2-dimensional ellipses separately in each reconstructed, non-attenuation corrected transaxial image slice

\section{Optimization of acquisition and reconstruction protocol}

The impact of angular sampling was tested with a custom-made 'star' phantom with two different sections as shown in Fig. 2. The total number of views $\left(n_{v}\right)$ acquired with the 3 detector heads was varied from 6 to 96, that is, the number of gantry positions $\left(n_{p}\right)$ was varied from 2 to $32\left(n_{P}=n_{v} / 3\right)$.

The SPECT system provides helical acquisition mode to avoid reconstruction artefacts due to insufficient axial sampling with the MPH collimators in circular acquisition mode (Meikle et al., 2014; Tuy, 1983). Helical acquisition is achieved by moving the patient table at each angular gantry step, that is, while the gantry moves from one angular position to the next, the table is moved by a distance $\Delta t_{d}$ in axial direction. The total displacement during the SPECT acquisition is $\mathrm{t}_{\mathrm{d}}=\mathrm{n}_{\mathrm{p}} * \Delta \mathrm{t}_{\mathrm{d}}$. The table moved out of the gantry during helical acquisition, because this simplifies fixing the start position of the scan (to avoid collision of the table and the detector heads). A custom-made multi-disk Defrise phantom (Fig. 3A) was used to test the impact of $\mathrm{t}_{\mathrm{d}}$ for helical acquisition mode for $\mathrm{t}_{\mathrm{d}}$ ranging from $0 \mathrm{~mm}$ to $138 \mathrm{~mm}$ (Bal et al., 2008; Gullberg et al., 1992). The distance between adjacent hot disks of the Defrise phantom was chosen to be $20 \mathrm{~mm}$, as this was considered a good compromise between (i) sufficiently large distance to avoid relevant spill-in from adjacent hot disks due to partial volume effects caused by limited spatial resolution in the reconstructed images and (ii) sufficiently small to safely detect the impact of variable axial sampling in MPH acquisition. Length and diameter of the Defrise phantom were intended to roughly match the CFOV of the MPH SPECT acquisition. The length was chosen somewhat larger than the axial length of the CFOV in circular acquisition mode $(120 \mathrm{~mm})$ in order to cover the CFOV also in helical acquisition mode with axial table motion. The diameter of the phantom was chosen slightly smaller than the diameter of the CFOV in order to safely avoid multiplexing (s. Fig. 3B). Interference between effects of axial sampling and effects of multiplexing might affect the optimization of table displacement for helical acquisition.

The 'optimal' number of views and the 'optimal' table displacement were selected by visual inspection of reconstructed high count MPH SPECT images of the star and the multi-disk phantom (pre-filter: none, default regularization predefined by the manufacturer, matrix size: 128 x 128, pixel size: $1.72 \mathrm{~mm}$ x 1.72 $\mathrm{mm}$, effective number of iterations (= number of iterations * number of subsets): 90 , number of subsets: 1 
to 3, CT-based attenuation and scatter correction, Monte Carlo quality: low, correction for radioactive decay during the scan).

Optimization of the reconstruction parameters for DAT SPECT in clinical routine was based on measurements of an anthropomorphic striatum phantom (Fig. 4A). The phantom was filled with different radioactivity solutions to achieve striatal contrast-to-background activity ratio of about 4:1 and 3:1 for left and right striatum, respectively. Caudate and putamen in one hemisphere were filled with the same radioactivity solution. Radioactivity concentration at the time of acquisition was $37.6 \mathrm{kBq} / \mathrm{ml}{ }^{99 \mathrm{~m}} \mathrm{Tc}$ in left caudate (volume $=5 \mathrm{ml})$ and putamen $(6 \mathrm{ml}), 28.2 \mathrm{kBq} / \mathrm{ml}$ in right caudate $(5 \mathrm{ml})$ and putamen $(6 \mathrm{ml})$, and $10 \mathrm{kBq} / \mathrm{ml}$ in the background $(1232 \mathrm{ml})$. Thus, total activity in the phantom was $13.0 \mathrm{MBq}$. This corresponds to $7.0-11.7 \%$ of the recommended dosage of $111-185 \mathrm{MBq}{ }^{123}$ I-FP-CIT for DAT SPECT in humans (Djang et al., 2012), in good agreement with the approximately $9 \%$ of injected activity distributing in the brain reported in studies of human biodistribution and dosimetry of ${ }^{123}$ I-FP-CIT (Booij et al., 1998). Thus, the activity in the anthropomorphic striatum phantom was representative of typical patient scans. The acquisition was performed with 30 gantry positions (90 views) each of $60 \mathrm{~s}$ duration resulting in a total acquisition duration of $30 \mathrm{~min}$ (also typical for clinical routine). The acquisition was performed in helical mode with total table displacement of $40 \mathrm{~mm}$. The following reconstruction parameters were tested: Monte Carlo quality: low, medium, high; effective number of iterations: 15, 33, 48, 66, 90, 150, 300, 498; number of subsets: $1,2,3,5$. Post reconstruction attenuation correction was performed according to Chang (Chang, 1978).

The 'optimal' reconstruction parameters were selected based on visual inspection of the reconstructed images of the anthropomorphic striatum phantom and on the contrast recovery coefficient (CRC) of the putamen (in PD, striatal DAT availability is most strongly effected in the putamen (Kaasinen and Vahlberg, 2017)). The CRC was computed as

$$
\mathrm{CRC}=\frac{\text { putamen to background ratio in the image }-1}{\text { true putamen to background ratio }-1}
$$

The mean count density in the unilateral putamen was obtained by hottest voxels analysis in large putamen ROIs predefined in a high resolution CT image of the striatum phantom (Fig. 4B) (Kupitz et al., 2014). The number of hottest voxels to be averaged was fixed to a total volume of $4.8 \mathrm{ml}$ for unilateral putamen 
corresponding to the volume of the putamen cavity as determined in the CT of the striatum phantom. The background was determined in a large reference region comprising the whole brain (excluding striata and spouts for phantom filling) with a safety margin to the edge of the brain in order to avoid partial volume effects (Fig. 4B). CRC analysis was performed fully automatically using a custom-made MATLAB script that first registered the SPECT image to the high-resolution CT and then applied the predefined ROIs.

\section{Count sensitivity}

The 3-dimensional system count sensitivity profile of the triple-head SPECT system with the MPH collimators was measured with a point source (about $10 \mathrm{MBq}{ }^{99 \mathrm{~m}} \mathrm{Tc}$ in about $5 \mu 1$ in an Eppendorf tube) placed on the lattice points of a $1 \mathrm{~cm}$ grid covering the whole FOV. A full SPECT acquisition was performed for each localization of the point source using the acquisition parameters optimized for clinical DAT SPECT as described in subsection "Optimization of acquisition and reconstruction protocol". The total number of counts acquired during the SPECT acquisition/was obtained by summing the counts over all views. Uniformity correction was turned on. Dead time correction was negligible (count rate below 10,000 counts per second in all measurements). The system count sensitivity at the localization of the point source was obtained by the following formula: sensitivity = total counts / total scan duration / activity of the point source decay-corrected to the start time of the SPECT acquisition.

The count sensitivity profile was measured for different values of total table displacement $t_{d}=0,40$, and $80 \mathrm{~mm}$ during helical scanning. In addition, peak sensitivity was measured for $\mathrm{t}_{\mathrm{d}}=0,20,40,60,80,100$, 120, $136 \mathrm{~mm}$.

For comparison, point source measurements for assessment of system sensitivity were also performed with the same triple-head camera equipped with low-energy-high-resolution-high-sensitivity (LEHRHS) collimators. The acquisitions with LEHRHS collimators were performed in double-head mode, that is, using only two of the three detectors. The third detector was switched off and moved away from the center of rotation in order to allow $14 \mathrm{~cm}$ radius of rotation with the two remaining detectors. The reason for this was that the minimum radius of rotation of the triple-head camera in conventional $120^{\circ}$ triple-head mode was $21 \mathrm{~cm}$, which is known to cause degradation of spatial resolution and contrast recovery (Koch et al., 2014). Interlaced triple-head mode (Kuikka et al., 1993) was not available for the triple-head camera used 
in this study. Acquisition parameters were as follows: total number of views $\mathrm{n}_{\mathrm{v}}=120$ (60 per head) resulting in $3^{\circ}$ angular sampling, scan arc $180^{\circ}$, matrix size $128 \times 128$, pixel size $=2.43 \mathrm{~mm}$.

Finally, point source measurements for assessment of system sensitivity were also performed with a double-head SPECT/CT system equipped with fan-beam collimators of $41 \mathrm{~cm}$ focal length/(Symbia TruePoint, Siemens Healthineers, Erlangen, Germany). A total number of 120 views was acquired (60 per head) for angular sampling of $3^{\circ}$. Projection data were sampled into a matrix of 128 x 128 pixels with 3.90 mm edge length. The scan arc was $180^{\circ}$.

The radius of rotation was $14 \mathrm{~cm}$ for all SPECT acquisitions independent of the SPECT system (triplehead with MPH, triple-head with LEHRHS in double-head mode, double-head system with fan-beam). Neither attenuation correction nor scatter correction was performed.

In order to test for potential differences in system count sensitivity of the triple-head SPECT with MPH collimators between ${ }^{99 \mathrm{~m}} \mathrm{Tc}$ and ${ }^{123} \mathrm{I}$, count sensitivity was measured also with a ${ }^{123} \mathrm{I}$ point source at several locations in the FOV.

\section{Spatial resolution}

Spatial resolution of MPH SPECT was assessed using a Derenzo type hot rod phantom with rod diameter of 2 to $7 \mathrm{~mm}$ filled with ${ }^{99 \mathrm{~m}} \mathrm{Tc}$ - or ${ }^{123} \mathrm{I}$-solution. In each segment, the rod diameter was equal to the minimum distance between the rods. Acquisition and reconstruction were performed using the protocols optimized for clinical DAT SPECT as described in subsection "Optimization of acquisition and reconstruction protocol”.

In order to further evaluate spatial resolution of MPH SPECT under clinical conditions, the following simulation was performed. Caudate nucleus, putamen and whole brain were segmented in a high resolution CT image of the anthropomorphic striatum phantom. Then voxel intensities were set to 4,3 and 1 for left striatum, right striatum, and rest of the brain, corresponding to the true contrasts in the MPH SPECT acquisition of the striatum phantom. Finally, the resulting image was filtered with Gaussian kernels of 3 , $4, \ldots, 8 \mathrm{~mm}$ full-width-at-half-maximum (FWHM) to simulate SPECT images with $3,4, \ldots, 8 \mathrm{~mm}$ spatial resolution. The simulated images were visually compared with the measured SPECT image of the striatum phantom reconstructed with the parameter settings optimized for clinical DAT SPECT. 
The point source measurements performed for characterization of the system sensitivity were used to test for potential non-uniformity of spatial resolution of MPH SPECT across the FOV. The MPH SPECT point source measurements were reconstructed with the reconstruction parameters optimized for clinical DAT SPECT. For comparison, the point source measurements acquired with LEHRHS or fan-beam collimators were reconstructed using reconstruction parameters that we had previously selected for clinical DAT SPECT with these collimators (LEHRHS collimators: filtered backprojection with Butterworth filter of order 6 and 0.55 cycles per pixel cutoff frequency, no post-filtering, matrix size 256 x 256 and pixel size $1.22 \mathrm{~mm}$; fan-beam collimators: ordered-subsets-expectation-maximization with resolution recovery implemented in the HybridRecon-Neurology tool of the Hermes SMART workstation v1.6 with parameter settings recommended for DAT SPECT by Hermes, that is, $128 \times 128$ pixels of $1.95 \mathrm{~mm}$ edge length, 16 iterations, 5 subsets, that is, 16 x $5=80$ effective iterations, resolution recovery with a Gaussian model (hole diameter $1.11 \mathrm{~mm}$, hole length $2.41 \mathrm{~cm}$, detector resolution $3.8 \mathrm{~mm}$, radius of rotation offset 3.21 $\mathrm{cm}$ ), and post-filtering with 3-dimensional Gaussian kernel of $7 \mathrm{~mm}$ FWHM).

Horizontal and vertical spatial resolution in the reconstructed point source images were obtained by fitting a Gaussian function to one-dimensional activity profiles obtained by first summing all transaxial image matrices and then summing over all rows or over all columns of the sum matrix. The mean of horizontal and vertical FWHM of the fitted Gaussian was used to characterize reconstructed spatial resolution at the location of the point source.

\section{Visual image quality and contrast recovery as function of the total counts}

The anthropomorphic striatum phantom with about 4:1 (left striatum) and 3:1 (right striatum) contrast and radioactivity concentrations representative of a typical patient study (left caudate and putamen: 37.6 $\mathrm{kBq} / \mathrm{ml}$, right caudate and putamen: $28.2 \mathrm{kBq} / \mathrm{ml}$, background: $10 \mathrm{kBq} / \mathrm{ml}$ ) was scanned with scan duration ranging from 2 minutes to about 2 hours using both the triple-head system with MPH collimators (optimized acquisition and reconstruction parameters) and the double-head system with fan-beam collimators (acquisition and reconstruction parameters as described in the previous subsection).

Post reconstruction Chang attenuation correction with broad-beam attenuation coefficient $\mu=0.12 / \mathrm{cm}$ was performed for all images (independent of the SPECT camera) using a custom-made MATLAB script in 
order to avoid bias by differences with respect to the attenuation correction method. No scatter correction was performed.

\section{Patient scan}

A male patient (54 y) referred to DAT SPECT for suspicion of PD in the early clinical phase was scanned twice after a single intravenous injection of $182 \mathrm{MBq}$ I-123-FP-CIT. The first scan of 40 min duration started $3 \mathrm{~h}$ 10min after tracer injection and used the triple-head camera equipped with LEHRHS collimators in double-head mode. The second scan of 30 min duration started $5 \mathrm{~h} 7 \mathrm{~min}$ after tracer injection and used the same triple-head camera equipped with MPH collimators in triple-head mode. Acquisition and reconstruction parameters with LEHRHS were as described above. Acquisition and reconstruction parameters with MPH were as optimized for DAT SPECT by the phantom measurements. Attenuation correction was performed by Chang's method implemented in the system software. 


\section{Results}

\section{Optimization of acquisition and reconstruction protocol}

Reconstructed images of the star phantom with varying angular sampling are shown in Figs. 2 B, D. Based on visual inspection of these images, $\mathrm{n}_{\mathrm{v}}=90$ was selected as the 'optimal' total number of views.

Reconstructed images of the multi-disk Defrise phantom with variable table displacement during helical scanning are shown in Fig. 3 B. Axial profiles of the reconstructed Defrise phantom with variable table displacement are shown in Fig. 5. Minimum total table displacement required to avoid clear image artefacts (blurriness) in the four central disks around the axial sensitivity peak of the MPH system (Fig. 3 B) and to avoid heterogeneity of peak height of those disks in the axial profile (Fig. 5) was $t_{d}=40 \mathrm{~mm}$. This value was selected as the 'optimal' total table displacement, because larger table displacement results in decreased peak count sensitivity (Fig. 8 E).

Results of the MPH SPECT measurements of the anthropomorphic striatum phantom for optimization of the reconstruction parameters are summarized in Figs. 6, 7. Based on these results, the following values were selected as 'optimal' compromise between image quality and reconstruction time: low Monte Carlo quality, 90 effective iterations, 3 subsets.

In summary, 'optimal' acquisition and reconstruction parameters for DAT SPECT with the triple-head SPECT system with MPH collimators were: $n_{v}=90, t_{d}=40 \mathrm{~mm}$, low Monte Carlo quality, 90 effective iterations, 3 subsets.

\section{Count sensitivity}

Profiles of system count sensitivity throughout the FOV of the triple-head system with the MPH collimators and the double-head system with fan-beam and LEHRHS collimators are shown in Fig. 8. Peak system sensitivity of the triple-head SPECT with MPH collimators was $620 \mathrm{cps} / \mathrm{MBq}$ compared to 225 cps/MBq for the double-head fan-beam system and $190 \mathrm{cps} / \mathrm{MBq}$ for the double-head LEHRHS system. Sensitivity of the MPH system decreased towards the edges of the FOV. The full width of the sensitivity profile at $200 \mathrm{cps} / \mathrm{MBq}$ was $11 \mathrm{~cm}$ in axial direction and $21 \mathrm{~cm}$ in transaxial direction (at the axial peak). 
The impact of varying table displacement for helical scanning on the axial sensitivity profile of the triplehead SPECT system with MPH collimators is shown in Fig. 8 E, F.

Peak count sensitivity of the triple-head system with the MPH collimators measured with ${ }^{123}$ I (rather than ${ }^{99 \mathrm{~m}} \mathrm{Tc}$ ) was $614 \mathrm{cps} / \mathrm{MBq}$ and $598 \mathrm{cps} / \mathrm{MBq}$ without and with $40 \mathrm{~mm}$ table displacement for helical scanning, respectively.

\section{Spatial resolution}

SPECT images of the Derenzo type hot rod phantom filled with ${ }^{99 m}$ Tc- or ${ }^{123}$ I-solution are shown in Fig. 9 $\mathrm{A}$ and $\mathrm{B}$, respectively. All rods with $\geq 5 \mathrm{~mm}$ diameter were clearly visible, all rods with $\leq 3 \mathrm{~mm}$ diameter could not be detected. Some of the $4 \mathrm{~mm}$ rods were visible, but with low contrast.

The simulated images of the anthropomorphic striatum phantom with varying spatial resolution are shown in Fig. 9 C. According to visual inspection, the simulated SPECT image with $5 \mathrm{~mm}$ spatial resolution shows best agreement with the measured MPH SPECT image (Fig. 9 B) with respect to spatial resolution.

The FWHM of the reconstructed point source images did not reveal relevant non-uniformity of spatial resolution of MPH SPECT across the central FOV (Fig. 8 B, D).

Visual image quality and contrast recovery as function of the total counts

Results of the MPH SPECT compared to fan-beam SPECT of the anthropomorphic striatum phantom for varying total number of counts are summarized in Figs. 10, 11.

\section{Patient scan}

Representative slices of the patient's I-123-FP-CIT SPECT with MPH collimators versus LEHRHS collimators are shown in Fig. 12. 


\section{Discussion}

The primary aim of the present study was to evaluate the performance of a triple-head SPECT camera with MPH collimators specifically designed for DAT SPECT to achieve (i) high peak sensitivity at the striatum for recovery of striatal tracer uptake with high spatial resolution and low statistical noise, and (ii) sufficiently broad sensitivity profile to cover the whole cerebrum to measure extrastriatal tracer uptake with adequate statistical quality for semi-quantitative analysis (reference region). In order to guarantee performance estimates obtained in the present study to be representative for DAT SPECT in everyday clinical patient care, acquisition and reconstruction protocol were optimized for clinical DAT SPECT using an anthropomorphic striatum phantom prior to the performance evaluation.

A major finding of the present study was the peak system sensitivity of the triple-head system with MPH collimators to be about 2.8 times higher than the sensitivity of a widely used double-head system with fanbeam collimators $(620 \mathrm{cps} / \mathrm{MBq}$ versus $225 \mathrm{cps} / \mathrm{MBq})$. Peak sensitivity per detector head was 207 cps/MBq and $113 \mathrm{cps} / \mathrm{MBq}$ for the MPH and for the fan-beam system, respectively. Thus, peak sensitivity of the MPH collimator was about $83 \%$ higher than the sensitivity of the fan-beam collimator. The improvement therefore was considerably larger than the $20-40 \%$ sensitivity gain provided by fan-beam collimators compared to parallel-hole collimators (Van Audenhaege et al., 2015a; Akiyama and Yui, 1991) which made practice guidelines on DAT SPECT recommend fan-beam collimators as the current state-ofthe-art for DAT SPECT (Darcourt et al., 2010; Djang et al., 2012).

The system sensitivity of the triple-head MPH SPECT was characterized by a profile with central peak and decreasing sensitivity towards the edges of the FOV, different from the uniform sensitivity of the doublehead SPECT system with fan-beam or LEHRHS collimators (Fig. 8). The axial MPH sensitivity profile was almost symmetrical around its peak, although the peak was not in the transaxial plane at the geometric center of the MPH collimator but was shifted by $7 \mathrm{~cm}$ towards the patient (Fig. $1 \mathrm{~A})$. Proper positioning of the patient's head (such that the striatum is located in the sensitivity peak) is required to make optimal use of the MPH collimator for DAT SPECT. The full width of the sensitivity profile at $200 \mathrm{cps} / \mathrm{MBq}$ (about the sensitivity of the double-head SPECT with fan-beam collimators) was $11 \mathrm{~cm}$ in axial direction and 21 $\mathrm{cm}$ in transaxial direction (at the axial peak) (Fig. $8 \mathrm{~A}, \mathrm{C}$ ). A study to optimize full-brain coverage in human brain MRI including 14,781 subjects (52\% male) covering the age range from 2 to 95 years reported 
the $99^{\text {th }}$ percentile of the linear dimension of the brain to be $143 \mathrm{~mm}$ in inferior-superior direction, $148 \mathrm{~mm}$ in left-right direction, and $190 \mathrm{~mm}$ in anterior-posterior direction (Mennes et al., 2014). Thus, the $21 \mathrm{~cm}$ transaxial FOV of the MPH SPECT is clearly sufficient for full transaxial coverage of the whole brain. Furthermore, helical acquisition mode with $40 \mathrm{~mm}$ total table displacement increases the axial FOV from 11 to $15 \mathrm{~cm}$. This is sufficient for full axial coverage of the brain in more than $99 \%$ of the patients (s. above). However, it requires accurate positioning of the patient's head to make sure that the brain is centered in the axial field-of-view. In SPECT/CT systems, this might be supported by a sagittal X-ray scout view. For SPECT-only systems, positioning lasers could be beneficial that indicate the center of rotation and the axial position of the sensitivity peak of the MPH collimators. Nevertheless, we recommend that next generation MPH collimators for DAT SPECT provide somewhat broader sensitivity profile in axial direction $(\geq 15 \mathrm{~cm}$ ). This would allow to use the same MPH collimator also for brain perfusion SPECT, in which inclusion of the whole brain including cerebellum is important (e.g., to detect crossed cerebellar diaschisis (Takasawa et al., 2002)).

Helical scanning not only extends the axial FOV but also improves image quality in the central FOV. For pinhole acquisition, Tuy's data completeness condition is only fulfilled in the plane described by the rotating focal point (the central slice) (Van Audenhaege et al., 2015a; Meikle et al., 2014; Tuy, 1983). In the MPH SPECT system evaluated in this study, Tuy's condition therefore is only fulfilled for the 4 transaxial slices defined by the orbit of the 4 transaxial pinhole rows. Helical sampling can reduce artefacts due to incomplete axial sampling (s. (Van Audenhaege et al., 2015a; Meikle et al., 2014) and references therein). With the MPH collimator evaluated in this study, minimum total table displacement during helical scanning required to avoid clear image artefacts was $40 \mathrm{~mm}$, close to the axial distance between the transaxial pinhole rows (Fig. 1 C). It should be noted that the beginning and the end of the axial FOV are poorly sampled in case of helical scanning. The total table displacement during helical scanning therefore should be large enough to avoid edge artefacts in the regions of interest.

A second major finding was the uniformity of the reconstructed spatial resolution of MPH SPECT across the central FOV (Fig. 8 B, D). Comparison of the high count SPECT image of the anthropomorphic striatum phantom with computer simulations of SPECT images with varying spatial resolution suggested a spatial resolution of about $5 \mathrm{~mm}$ FWHM for MPH SPECT with the acquisition and reconstruction protocol optimized for clinical DAT SPECT (Fig. 9). A limitation of this simulation experiment to estimate 
spatial resolution of MPH SPECT under clinical conditions was that the simulation did not include attenuation nor scatter. As a consequence, potential residual attenuation and scatter effects in the measured SPECT image of the anthropomorphic striatum phantom might have affected the estimation of spatial resolution of clinical MPH SPECT. In order to reduce this risk, CT-based correction had been used to correct the measured SPECT for attenuation and scatter, because this is more accurate than post reconstruction Chang attenuation correction. Furthermore, attenuation and scatter mainly effect the low frequency components of the SPECT image. The impact of attenuation and scatter on the high frequency components of the image that determine spatial resolution is much smaller. A clinical MPH SPECT system (G-SPECT-I) with full angular coverage was reported to provide 2.5 to $3.5 \mathrm{~mm}$ spatial resolution (Beekman et al., 2015; Chen et al., 2018).

Measurements of the anthropomorphic striatum phantom with varying scan duration confirmed superior sensitivity and spatial resolution of MPH SPECT compared to fan-beam SPECT (Figs. 10, 11). With the same phantom filling, the triple-head MPH system acquired about $65 \%$ more counts per minute than the double-head fan-beam system. The difference is smaller than the $180 \%$ difference between peak sensitivity of the MPH system and uniform sensitivity of the fan-beam system, because the total number of counts obtained by MPH SPECT of the striatum phantom is proportional to the average of the MPH sensitivity over all locations covered by the phantom weighted by the local activity concentration. Higher spatial resolution of MPH SPECT compared to fan-beam SPECT resulted in better anatomical delineation of the striata in the reconstructed images (Fig. 10) and higher contrast recovery (Fig. 11). Image quality reached a plateau at about 5 million counts for both systems. The total scan duration to acquire 5 million counts from the striatum phantom with realistic activity concentration was $32 \mathrm{~min}$ for the triple-head MPH system versus 54 min for the double-head fan-beam system.

Superior imaging characteristics of MPH DAT SPECT compared to fan-beam DAT SPECT was confirmed in a patient scan (Fig, 12). Image quality of the MPH DAT SPECT in the patient was almost PET-like, although acquisition time was shorter compared to fan-beam SPECT (30 min versus $40 \mathrm{~min}$ ).

Some procedure guidelines for DAT SPECT recommend that visual interpretation of the SPECT images is complemented by semi-quantitative analysis of FP-CIT uptake in the striatum and its sub-regions (Darcourt et al., 2010). However, striatal FP-CIT uptake in the reconstructed images is sensitive to camera- 
specific differences of acquisition and reconstruction protocol, which limits the use of semi-quantitative analysis in DAT SPECT. In particular, camera-specific variability of spatial resolution causes variable underestimation of striatal FP-CIT uptake by up to 50\% (Lange et al., 2014b). This complicates sharing of DAT SPECT normal databases and cutoff values for semi-quantitative measures between sites and/or SPECT cameras. Thus, knowing the "true" value of striatal tracer binding in DAT SPECT would have direct benefit, namely the transferability of the result across cameras and sites. Follow-up DAT SPECT (Apostolova et al., 2017) using different cameras would also benefit if true semi-quantitative values could be provided every time. MPH DAT-SPECT is a step towards the true value, as was shown by improved CRC of MPH SPECT (Fig. 11 A).

The utility of MPH collimators for DAT SPECT in humans has been investigated previously by several groups. King and co-workers proposed a combination of a MPH collimator on one head of a double-head SPECT system and a fan-beam collimator on the other head (King et al., 2016). The rationale was that the MPH collimator would provide high resolution of $4.8 \mathrm{~mm}$ (Konik et al., 2018) and high sensitivity for imaging of the interior portion of the brain, particularly the striatum. The fan-beam collimator would provide lower resolution, but complete sampling of the brain addressing data sufficiency and allowing a volume-of-interest to be defined over cortical brain reference regions for semi-quantitative analysis (King et al., 2016). Lee and co-workers demonstrated that MPH collimators can provide an over an order of magnitude enhancement in peak count sensitivity compared to conventional parallel-hole collimators (Lee et al., 2014). Neither the system proposed by King and co-workers nor the system proposed by Lee and co-workers has yet been made available for widespread clinical use. Beekman and co-workers developed a clinical MPH SPECT system (G-SPECT-I) with full angular coverage by stationary detectors and adaptable bore size for improved resolution-sensitivity tradeoff (Beekman et al., 2015). For SPECT imaging of the human brain, the system provides $2.5 \mathrm{~mm}$ spatial resolution with about $400 \mathrm{cps} / \mathrm{MBq}$ sensitivity or $3.5 \mathrm{~mm}$ spatial resolution with about $900 \mathrm{cps} / \mathrm{MBq}$ (Beekman et al., 2015; Chen et al., 2018). Recently, the acquisition protocol for DAT-SPECT with this system has been optimized (Chen et al., 2018).

The triple-head SPECT system evaluated in the present study is a conventional general-purpose SPECT system that can also be operated with conventional collimators. Three heads not only provide $50 \%$ higher count sensitivity compared to two heads, but also allow for more flexibility in the MPH-design with respect to the combination of multi-pinhole collimators with other collimator types, e.g. fan-beam collimators as 
proposed by King and co-workers (King et al., 2016). In addition, 3 heads provide better potential for stationary acquisition (without detector motion) with MPH collimators for fast dynamic imaging (Lange et al., 2014a).

A limitation of the present study is that most phantom measurements were performed with ${ }^{99 \mathrm{~m}} \mathrm{Tc}$ rather than ${ }^{123} \mathrm{I}$ in order to save costs. However, within the limits of accuracy of the measurements, MPH peak sensitivity did not differ between ${ }^{99 \mathrm{~m}} \mathrm{Tc}$ and ${ }^{123} \mathrm{I}$, suggesting that results and conclusions of the present study can be translated to DAT SPECT with ${ }^{123}$ I-FP-CIT. This was confirmed by the ${ }^{123}$ I-FP-CIT DAT SPECT in a patient (Fig. 12). A further limitation of this study is that the diameter of the custom-made multi-disk Defrise phantom used to test the impact of table displacement during helical acquisition was slightly smaller than the diameter of the central field-of-view (113 mm versus $120 \mathrm{~mm})$. This most likely resulted in underestimation of the effect of axial undersampling compared to objects with larger diameter. 


\section{Conclusion}

MPH collimators provide considerable improvement of image quality in DAT SPECT with respect to both spatial resolution and statistical noise compared to conventional collimators including low-energy-highresolution-high-sensitivity collimators and fan-beam collimators. As visual interpretation of DAT SPECT performed with conventional collimators by experienced readers already provides high diagnostic accuracy ( $\geq 90 \%$ sensitivity and specificity (O'Brien et al., 2014)), we expect improved image quality in MPHDAT SPECT to be particularly useful in borderline cases and for less experienced readers. We also expect it to result in improved reader confidence independent of the reader's experience. However, the largest benefit of improved image quality in DAT SPECT by MPH technology is expected for early detection (or exclusion) of PD before the loss of putaminal DAT reaches 50\% and PD-characteristic motor symptoms occur. These hypotheses might be tested in prospective clinical studies, preferably by comparing MPH SPECT and parallel-hole/fan-beam SPECT acquired in randomized order after a single injection of FPCIT in the same patients.

\section{Potential conflicts of interest}

K. Tecklenburg has previously been part-time employee of Mediso GmbH, Germany, a subsidiary company of Mediso Medical Imaging Systems, Budapest, Hungary, the manufacturer of the multi-pinhole collimator evaluated in this study. A. Forgács is full-time employee of Scanomed Nuclear Medicine Center Debrecen, Hungary, a subsidiary company of Mediso Medical Imaging Systems. J. Csirik has worked as consultant of Mediso Medical Imaging Systems. However, this did not bias their work, as they have no financial interest in the results of the present study. There is no actual or potential conflict of interest for any of the other authors. 


\section{References}

2017 NICE guideline [NG71]: Parkinson's disease in adults. National Institute for Health Care and Excellence)

Akiyama Y and Yui N 1991 Performance of a multislice fan beam collimator for SPECT imaging of the head Ann Nucl Med 5 117-21

Alvarez-Fischer D, Blessmann G, Trosowski C, Béhé M, Schurrat T, Hartmann A, Behr T M, Oertel W H Höglinger G U and Höffken H 2007 Quantitative [(123)I]FP-CIT pinhole SPECT imaging predicts striatal dopamine levels, but not number of nigral neurons in different mouse models of Parkinson's disease. NeuroImage 38 5-12

Andringa G, Drukarch B, Bol J G, de Bruin K, Sorman K, Habraken J B and Booij J 2005 Pinhole SPECT imaging of dopamine transporters correlates with dopamine transporter immunohistochemical analysis in the MPTP mouse model of Parkinson's disease Neuroimage 26 1150-8

Apostolova I, Niedzielska D, Derlin T, Koziolek E J, Amthauer H, Salmen B, Pahnke J, Brenner W, Mautner V F and Buchert R 2015 Perfusion single photon emission computed tomography in a mouse model of neurofibromatosis type 1: towards a biomarker of neurologic deficits J Cerebr Blood F Met 35 1304-12

Apostolova I, Taleb D S, Lipp A, Galazky I, Kupitz D, Lange C, Makowski M R, Brenner W, Amthauer H, Plotkin M and Buchert R 2017 Utility of Follow-up Dopamine Transporter SPECT With 123IFP-CIT in the Diagnostic Workup of Patients With Clinically Uncertain Parkinsonian Syndrome Clin Nucl Med 42 589-94

Apostolova I, Wunder A, Dirnagl U, Michel R, Stemmer N, Lukas M, Derlin T, Gregor-Mamoudou B, Goldschmidt J, Brenner W and Buchert R 2012 Brain perfusion SPECT in the mouse: Normal pattern according to gender and age Neuroimage 63 1807-17

Azazrm A, Gharapapagh E, Islamian J and Mahmoudian B 2015 Advances in Pinhole and Multi-Pinhole Collimators For Single Photon Emission Computed Tomography Imaging World Journal of Nuclear Medicine 143

Bae J, Bae S, Lee S-y, Lee K, Kim Y, Joung J, Kim, M and Kim K M 2017 Two-level multi-pinhole collimator for SPECT imaging using a small-field-of-view gamma camera Journal of the Korean Physical Society 70 192-200

Bal G, Acton P D, Jansen F and Hasegawa B H 2008 Revolving multipinhole SPECT for small animal imaging. In: 2008 IEEE Nuclear Science Symposium Conference Record: IEEE) pp 5577-84

Beekman F and Van Der Have F 2007 The pinhole: Gateway to ultra-high-resolution three-dimensional radionuclide imaging Eur J Nucl Med Mol I 34 151-61

Beekman F J, van der Have F, Goorden M C, Vaissier P E B, van Roosmalen J, During H and Vastenhouw B 2015 G -SPECT-I: a full ring high sensitivity and ultra-fast clinical molecular imaging system with < 3mm resolution Eur J Nucl Med Mol I 42 S209-S

Beekman F J, van der Have F, Vastenhouw B, van der Linden A J, van Rijk P P, Burbach J P and Smidt M P 2005 U-SPECT-I: a novel system for submillimeter-resolution tomography with radiolabeled molecules in mice J Nucl Med 46 1194-200

Berardelli A, Wenning G K, Antonini A, Berg D, Bloem B R, Bonifati V, Brooks D, Burn D J, Colosimo C, Fanciulli A, Ferreira J, Gasser T, Grandas F, Kanovsky P, Kostic V, Kulisevsky J, Oertel W, Poewe W, Reese J P, Relja M, Ruzicka E, Schrag A, Seppi K, Taba P and Vidailhet M 2013 EFNS/MDS-ES/ENS [corrected] recommendations for the diagnosis of Parkinson's disease Eur J Neurol 20 16-34

Bernheimer H, Birkmayer W, Hornykiewicz O, Jellinger K and Seitelberger F 1973 Brain dopamine and the syndromes of Parkinson and Huntington. Clinical, morphological and neurochemical correlations J Neurol Sci 20 415-55 
Booij J, Sokole E B, Stabin M G, Janssen A G M, de Bruin K and van Royen E A 1998 Human biodistribution and dosimetry of [I-123]FP-CIT: a potent radioligand for imaging of dopamine transporters Eur J Nucl Med 25 24-30

Buchert R, Buhmann C, Apostolova I, Meyer P T and Gallinat J 2019 Nuclear Imaging in the Diagnosis of Clinically Uncertain Parkinsonian Syndromes Dtsch Arztebl Int 116 747-54

Buvat I and Castiglion I 2002 Monte Carlo simulations in SPET and PET Q J Nucl Med 46 48-61

Cao Z, Bal G, Accorsi R and Acton P D 2005 Optimal number of pinholes in multi-pinhole SPECT for mouse brain imaging — a simulation study Physics in Medicine and Biology 50 4609-24

Chahine L M, Iranzo A, Fernandez-Arcos A, Simuni T, Seedorff N, Caspell-Garcia C, Amara A W, Comella C, Hogl B, Hamilton J, Marek K, Mayer G, Mollenhauer B, Postuma R, Tolosa E, Trenkwalder C, Videnovic A, Oertel W and Group P S W 2019 Basic clinical features do not predict dopamine transporter binding in idiopathic REM behavior disorder NPJ Parkinşons Dis 5 2

Chang L T 1978 Method for Attenuation Correction in Radionuclide Computed Tomography Ieee T Nucl Sci 25 638-43

Chen L, Tsui B M W and Mok G S P 2017 Design and evaluation of two multi-pinhole collimators for brain SPECT Annals of Nuclear Medicine 31 636-48

Chen Y, Vastenhouw B, Wu C, Goorden M C and Beekman F J 2018 Optimized image acquisition for dopamine transporter imaging with ultra-high resolution elinical pinhole SPECT Phys Med Biol 63225002

Darcourt J, Booij J, Tatsch K, Varrone A, Borght T V, Kapucu O L, Nagren K, Nobili F, Walker Z and Van Laere K 2010 EANM procedure guidelines for brain neurotransmission SPECT using I-123labelled dopamine transporter ligands, version 2 Eur J Nucl Med Mol I 37 443-50

Difilippo F P 2008 Design and performance of a multi-pinhole collimation device for small animal imaging with clinical SPECT and SPECT-CT scanners Phys Med Biol 53 4185-201

Djang D S, Janssen M J, Bohnen N, Booij J, Henderson T A, Herholz K, Minoshima S, Rowe C C, Sabri O, Seibyl J, Van Berckel B N and Wanner M 2012 SNM practice guideline for dopamine transporter imaging with 123I-ioflupane SPECT 1.0 J Nucl Med 53 154-63

Fearnley J M and Lees A J 1991 Ageing and Parkinson's disease: substantia nigra regional selectivity Brain 114 ( Pt 5) 2283-301

Geisler S, Beindorff N, Cremer M, Hoffmann K, Brenner W, Cumming P, Meyer P T, Langen K J, Fuchs E and Buchert R 2015 Characterization of [I-123]FP-CIT binding to the dopamine transporter in the striatum of tree shrews by quantitative in vitro autoradiography Synapse 69 497-504

Gibb W R G and Lees A J 1988 The Relevance of the Lewy Body to the Pathogenesis of Idiopathic Parkinsons-Disease J Neurol Neurosur Ps 51 745-52

Green P J 1990 Bayesian Reconstructions from Emission Tomography Data Using a Modified Em Algorithm Ieee Transactions on Medical Imaging 9 84-93

Gullberg G T, Zeng G L, Datz F L, Christian P E, Tung C H and Morgan H T 1992 Review of Convergent Beam Tomography in Single Photon-Emission Computed-Tomography Physics in Medicine and Biology 37 507-34

Iranzo A, Santamaria J, Valldeoriola F, Serradell M, Salamero M, Gaig C, Ninerola-Baizan A, SanchezValle R, Llado A, De Marzi R, Stefani A, Seppi K, Pavia J, Hogl B, Poewe W, Tolosa E and Lomena F 2017 Dopamine transporter imaging deficit predicts early transition to synucleinopathy in idiopathic rapid eye movement sleep behavior disorder Ann Neurol 82 419-28

Ivashchenko O, van der Have F, Villena J L, Groen H C, Ramakers R M, Weinans H H and Beekman F J 2014 Quarter-millimeter-resolution molecular mouse imaging with U-SPECT(+) Mol Imaging 13

Johnson L C, Moore S C and Metzler S D 2016 Effect of pinhole shape on projection resolution Physics in Medicine and Biology 61 2003-13 
Kaasinen V and Vahlberg T 2017 Striatal dopamine in Parkinson disease: A meta-analysis of imaging studies Ann Neurol 82 873-82

Kim K S 2017 Toward neuroprotective treatments of Parkinson's disease Proc Natl Acad Sci U S A 114 3795-7

King M A, Mukherjee J M, Konik A, Zubal I G, Dey J and Licho R 2016 Design of a Multi-Pinhole Collimator for I-123 DaTscan Imaging on Dual-Headed SPECT Systems in Combination with a Fan-Beam Collimator Ieee T Nucl Sci 63 90-7

Koch W, Bartenstein P and la Fougere C 2014 Radius dependence of FP-CIT quantification: a Monte Carlo-based simulation study Ann Nucl Med 28 103-11

Konik A, De Beenhouwer J, Mukherjee J M, Kalluri K, Banerjee S, Zeraatkar N, Fromme T and King M A 2018 Simulations of a Multi-Pinhole SPECT Collimator for Clinical Dopamine Transporter (DAT) Imaging IEEE Trans Radiat Plasma Med Sci 2 444-51

Kuikka J T, Tenhuneneskelinen M, Jurvelin J and Kiilianen H 1993 Physical Performance of the Siemens Multispect 3-Gamma-Camera Nuclear Medicine Communications 14 490-7

Kupitz D, Apostolova I, Lange C, Ulrich G, Amthauer H, Brenner W and Buchert R 2014 Global scaling for semi-quantitative analysis in FP-CIT SPECT Nuklearmedizin 53 234-41

Lange C, Apostolova I, Lukas M, Huang K P, Hofheinz F, Gregor-Mamoudou B, Brenner W and Buchert R 2014a Performance Evaluation of Stationary and Semi-Stationary Acquisition with a NonStationary Small Animal Multi-Pinhole SPECT System Mol Imaging Biol 16 311-6

Lange C, Seese A, Schwarzenbock S, Steinhoff K, Umland-Seidler B, Krause B J, Brenner W, Sabri O, Kurth J, Hesse S and Buchert R 2014b CT-Based Attenuation Correction in I-123-Ioflupane SPECT Plos One 9

Lee T C, Ellin J R, Huang Q, Shrestha U, Gullberg G T and Seo Y 2014 Multipinhole collimator with 20 apertures for a brain SPECT application Med Phys 41

Lin J Y 2013 On Artifact-Free Projection Overlaps in Multi-Pinhole Tomographic Imaging Ieee Transactions on Medical Imaging 32 2215-29

Meikle S R, Kench P L and Lin J 2014 Molecular Imaging of Small Animals: Instrumentation and Application, ed H Zaidi (New York: Springer)

Meles S K, Vadasz D, Renken R J, Sittig-Wiegand E, Mayer G, Depboylu C, Reetz K, Overeem S, Pijpers A, Reesink F E, van Laar T, Heinen L, Teune L K, Hoffken H, Luster M, Kesper K, Adriaanse S M, Booij J, Leenders K L and Oertel W H 2017 FDG PET, dopamine transporter SPECT, and olfaction: Combining biomarkers in REM sleep behavior disorder Mov Disord 32 1482-6

Mennes M, Jenkinson M, Valabregue R, Buitelaar J K, Beckmann C and Smith S 2014 Optimizing fullbrain coverage in human brain MRI through population distributions of brain size Neuroimage 98 513-20

Nakabayashi S, Chikamatsu T, Okamoto T, Kaminaga T, Arai N, Kumagai S, Shiraishi K, Okamoto T, Kobayashi T and Kotoku J 2018 Denoising Projection Data with a Robust Adaptive Bilateral Filter in Low-Count SPECT International Journal of Medical Physics, Clinical Engineering and Radiation Oncology 7 363-75

Nillius P and Danielsson M 2010 Theoretical bounds and system design for multipinhole SPECT IEEE Transactions on Medical Imaging 29 1390-400

Niznik H B, Fogel EF, Fassos F F and Seeman P 1991 The dopamine transporter is absent in parkinsonian putamen and reduced in the caudate nucleus J Neurochem 56 192-8

Nuyts J, Vunckx K, Defrise M and Vanhove C 2009 Small animal imaging with multi-pinhole SPECT Methods 48 83-91

O'Brien J T, Oertel W H, McKeith I G, Grosset D G, Walker Z, Tatsch K, Tolosa E, Sherwin P F and Grachev I D 2014 Is ioflupane I123 injection diagnostically effective in patients with movement disorders and dementia? Pooled analysis of four clinical trials Bmj Open 4 
Panin V Y, Zeng G L and Gullberg G T 1999 Total variation regulated EM algorithm Ieee T Nucl Sci 46 2202-10

Peterson T E and Shokouhi S 2012 Advances in Preclinical SPECT Instrumentation Journal of Nuclear Medicine 53 841-4

Rentmeester M C M, Van Der Have F and Beekman F J 2007 Optimizing multi-pinhole SPECT geometries using an analytical model Physics in Medicine and Biology 52 2567-81

Salvado D, Erlandsson K, Bousse A, Occhipinti M, Busca P, Fiorini C and Hutton B F 2015 Collimator Design for a Brain SPECT/MRI Insert Ieee T Nucl Sci 62 1716-24

Scherfler C, Donnemiller E, Schocke M, Dierkes K, Decristoforo C, Oberladstätter M, Kolbitsch C, Zschiegner F, Riccabona G, Poewe W and Wenning G 2002 Evaluation of striatal dopamine transporter function in rats by in vivo beta-[123I]CIT pinhole SPECT. NeuroImage 17 128-41

Shokouhi S, Metzler S D, Wilson D W and Peterson T E 2009 Multi-pinhole collimator design for smallobject imaging with SiliSPECT: A high-resolution SPECT Physics in Medicine and Biology 54 207-25

Si C, Mok G S P, Chen L and Tsui B M W 2016 Design and evaluation of an adaptive multipinhole collimator for high-performance clinical and preclinical imaging Nuclear Medicine Communications 37 313-21

Spilker M E, Bal G, Uribe J, Henderson D, Thurfjell L, Hehir C T, Tao X, Can A, Sarachan B and Jansen F 2008 Evaluation of different multi-pinhole imaging geometries for SPECT imaging of parkinsonian disorders IEEE Nuclear Science Symposium Conference Record 4022-4

Takasawa M, Watanabe M, Yamamoto S, Hoshi T, Sasaki T, Hashikawa K, Matsumoto M and Kinoshita N 2002 Prognostic value of subacute crossed cerebellar diaschisis: single-photon emission CT study in patients with middle cerebral artery territory infarct AJNR Am J Neuroradiol 23 189-93

Tuy H K 1983 An Inversion-Formula for Cone-Beam Reconstruction Siam J Appl Math 43 546-52

Twelves D, Perkins K S and Counsell C 2003 Systematic review of incidence studies of Parkinson's disease Mov Disord 18 19-31

Vaissier P E, Goorden M C, Vastenhouw B, van der Have F, Ramakers R M and Beekman F J 2012 Fast spiral SPECT with stationary gamma-cameras and focusing pinholes J Nucl Med 53 1292-9

Van Audenhaege K, Van Holen R, Vandenberghe S, Vanhove C, Metzler S D and Moore S C 2015a Review of SPECT collimator selection, optimization, and fabrication for clinical and preclinical imaging Med Phys 42 4796-813

Van Audenhaege K, Vanhove C, Vandenberghe S and Van Holen R 2015b The Evaluation of Data Completeness and Image Quality in Multiplexing Multi-Pinhole SPECT Ieee Transactions on Medical Imaging 34 474-86

Vanhove C, Defrise M, Lahoutte T and Bossuyt A 2008 Three-pinhole collimator to improve axial spatial resolution and sensitivity in pinhole SPECT Eur J Nucl Med Mol I 35 407-15

Vastenhouw B, van der Have F, van der Linden A J, von Oerthel L, Booij J, Burbach J P, Smidt M P and Beekman F J 2007 Movies of dopamine transporter occupancy with ultra-high resolution focusing pinhole SPECT Mol Psychiatry 12 984-7

Vunckx K, Nuyts J, Vanbilloen B, De Saint-Hubert M, Vanderghinste D, Rattat D, Mottaghy F M and Defrise M 2009 Optimized multipinhole design for mouse imaging Ieee T Nucl Sci 56 2696-705

Woodcock E, Murphy T, Hemmings P and Longworth S 1965 Techniques used in the GEM code for Monte Carlo neutronics calculations in reactors and other systems of complex geometry. In: Applications of Computing Methods to Reactor Problems, (Lemont, IL, USA: Argonne National Laboratories Report ANL-7050)

Yan P, Chen L, Tsui B M W and Mok G S P 2016 Evaluation of Stationary and Semi-stationary Acquisitions from Dual-head Multi-pinhole Collimator for Myocardial Perfusion SPECT Journal of Medical and Biological Engineering 36 675-85 


\section{A}

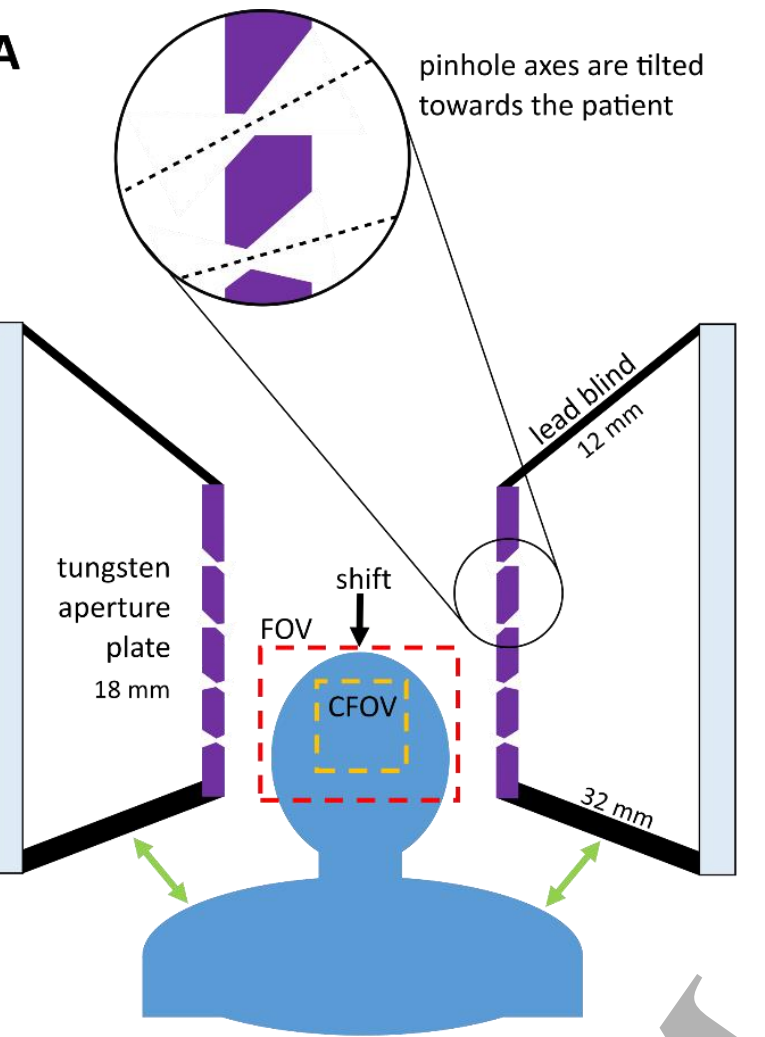

B

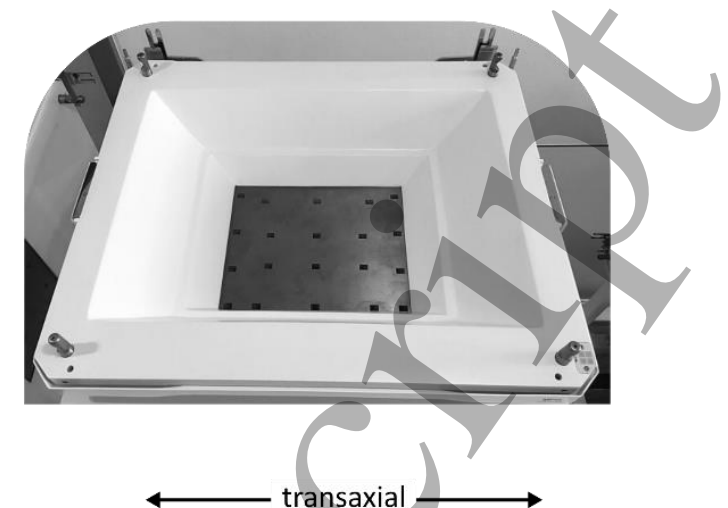

C

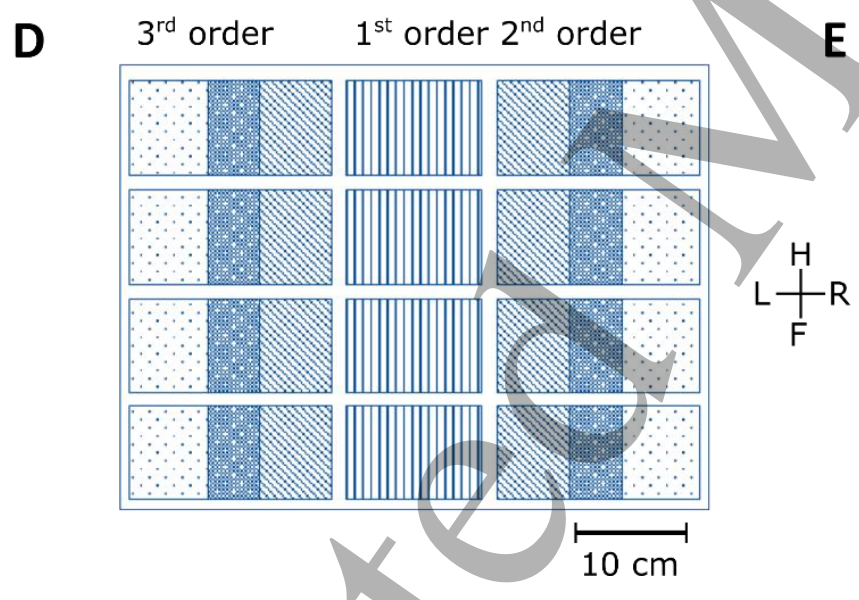

E $3^{\text {rd }}$ order $\quad 1^{\text {st }}$ order $2^{\text {nd }}$ order

Figure 1 (A) Drawing of the detector heads equipped with the multi-pinhole collimator (only two of the three detector heads are shown). The pinhole axes are tilted towards the patient such that the field-of-view (FOV) is shifted $7 \mathrm{~cm}$ towards the patient in axial direction. This simplifies positioning the striatum in the central FOV (CFOV) also in patients with short neck. Part (B) shows a photograph of the rear view of the MPH collimator. Part (C) is a drawing showing the arrangement of the 20 pinholes on the tungsten aperture plate. Part (D) is a drawing of the projection pattern on the scintillation crystal to illustrate the combination 
of non-overlapping and overlapping projections. Part (E) shows the projection pattern of a ${ }^{57}$ Co flood phantom placed directly on the touch plate on top of the aperture plate. 

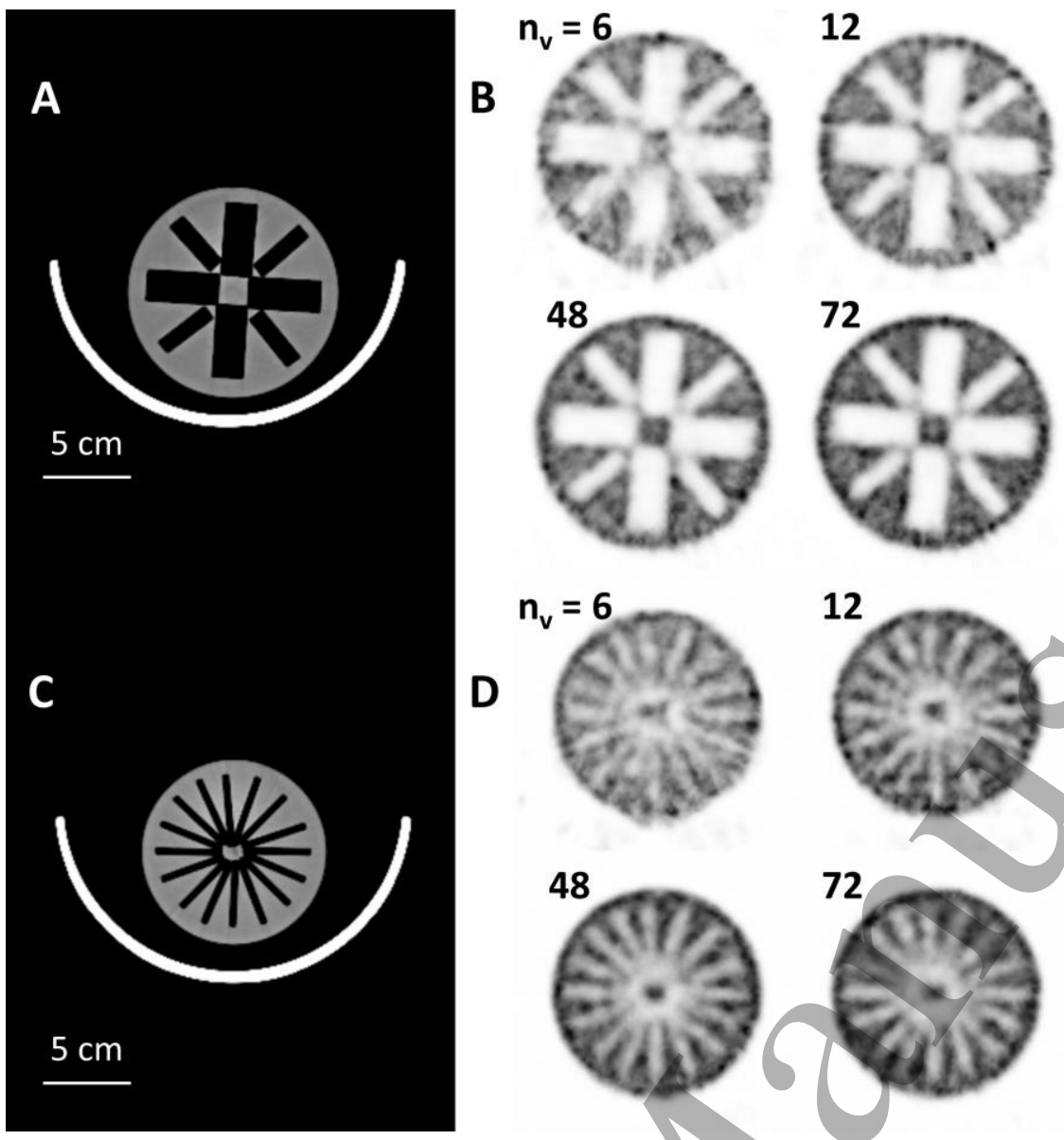

24

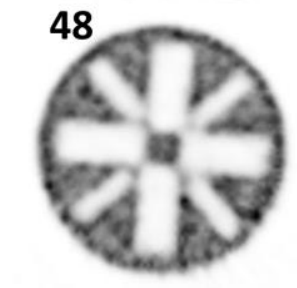

72
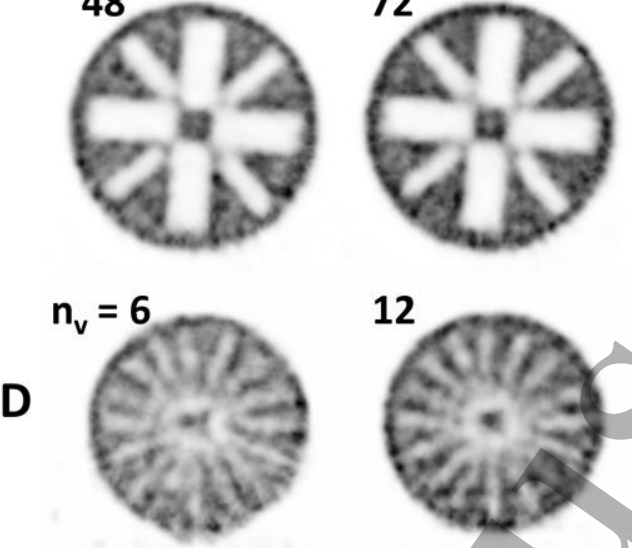

12
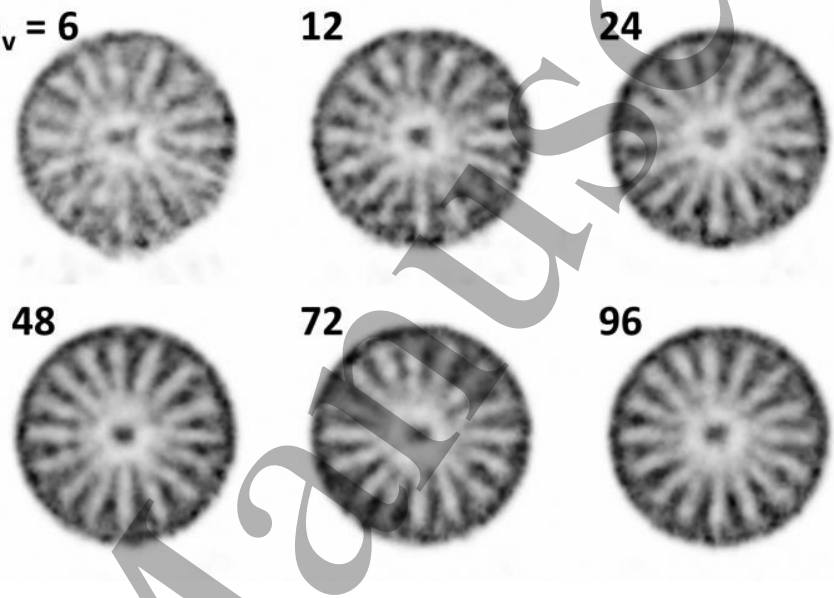

Figure 2 Transaxial CT slices (A, C) through the two sections of a custom-made star phantom used to optimize angular sampling of MPH SPECT, and transaxial MPH SPECT images obtained with varying total number $\mathrm{n}_{\mathrm{v}}$ of views $(\mathbf{B}, \mathbf{D})$. Total acquisition time of the star phantom was $16 \mathrm{~min}$, independent of the total number of views. The total number of counts varied between 61 and 79 million (due to radioactive decay between the scans). 


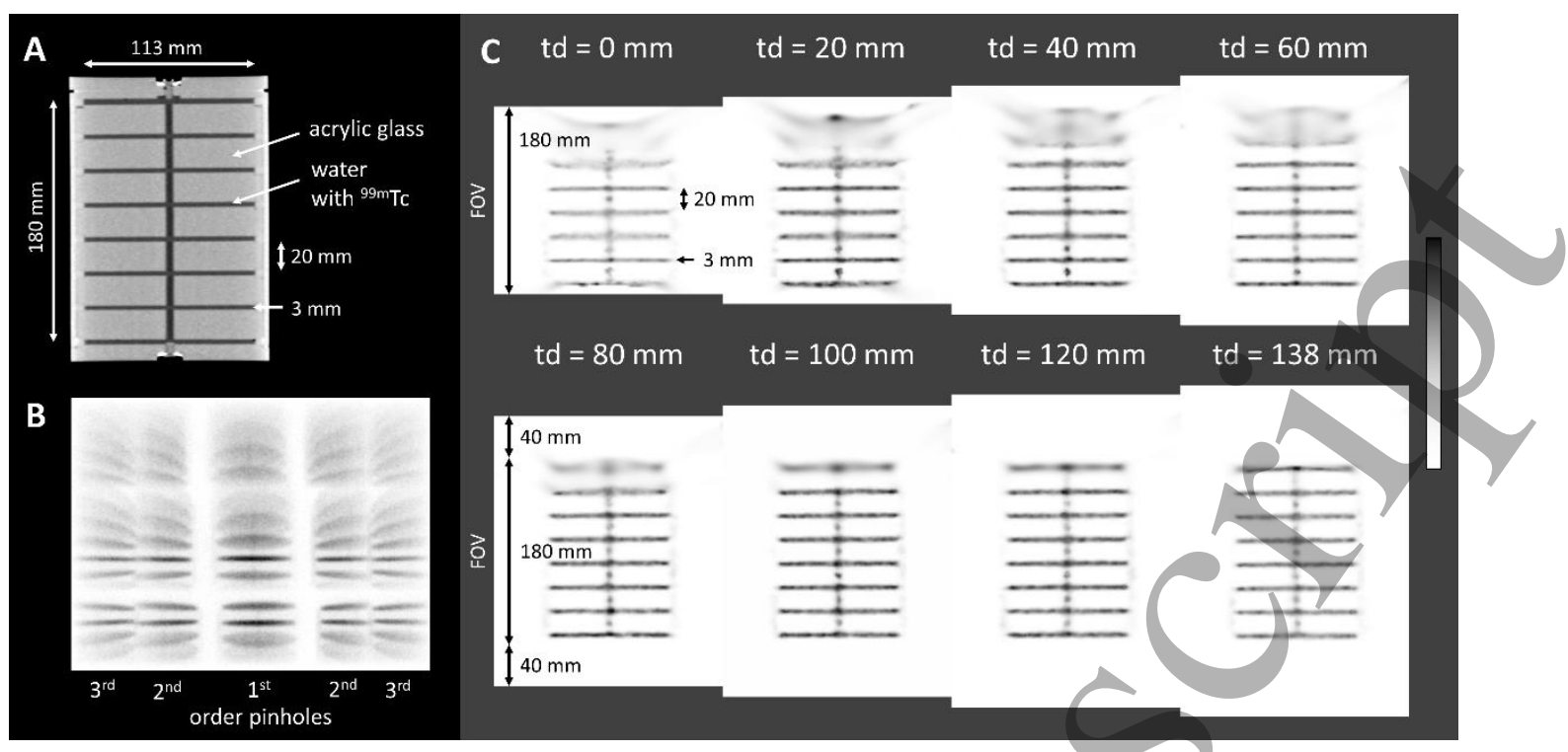

Figure 3 Central sagittal CT slice through the custom-made multi-disk Defrise phantom used for optimization of total table displacement $t_{d}$ during helical MPH scanning (A), its MPH projection on the detector (B), and a central sagittal slice of the reconstructed MPH SPECT images acquired in circular $\left(\mathrm{t}_{\mathrm{d}}\right.$ $=0)$ and multiple helical modes $\left(t_{d}>0\right)(\mathbf{C})$. The length of the Defrise phantom (= distance between the two outer disks) was $180 \mathrm{~mm}$, its inner diameter (= diameter of the hot disks) was $113 \mathrm{~mm}$. Helical acquisition extends the axial FOV (by $t_{d}$ ), which explains the increasing size of the sagittal SPECT slice with increasing $t_{d}$. All other acquisition and reconstruction parameters (except $t_{d}$ ) were kept fixed. CTbased attenuation and scatter correction was performed. The color map was adjusted separately for each SPECT image. 


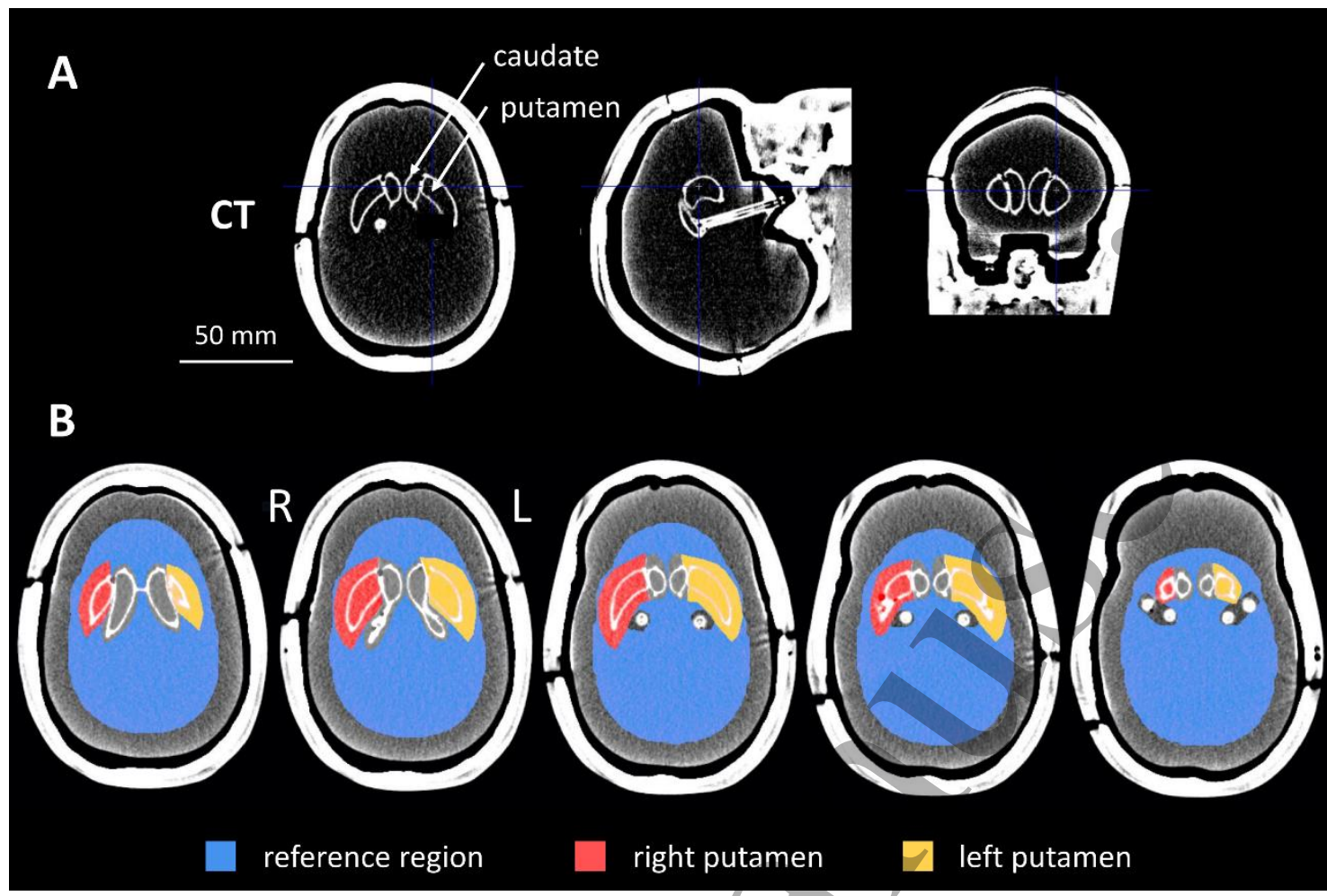

Figure 4 (A): Orthogonal CT slices through the anthropomorphic striatum phantom used to optimize MPH image reconstruction settings for DAT SPECT in clinical routine. (B): Large predefined regions of interest for hottest voxels analysis of right (red) and left (yellow) putamen and for the reference region (blue) used as background for computation of the putaminal contrast recovery coefficient in the reconstructed SPECT images. 

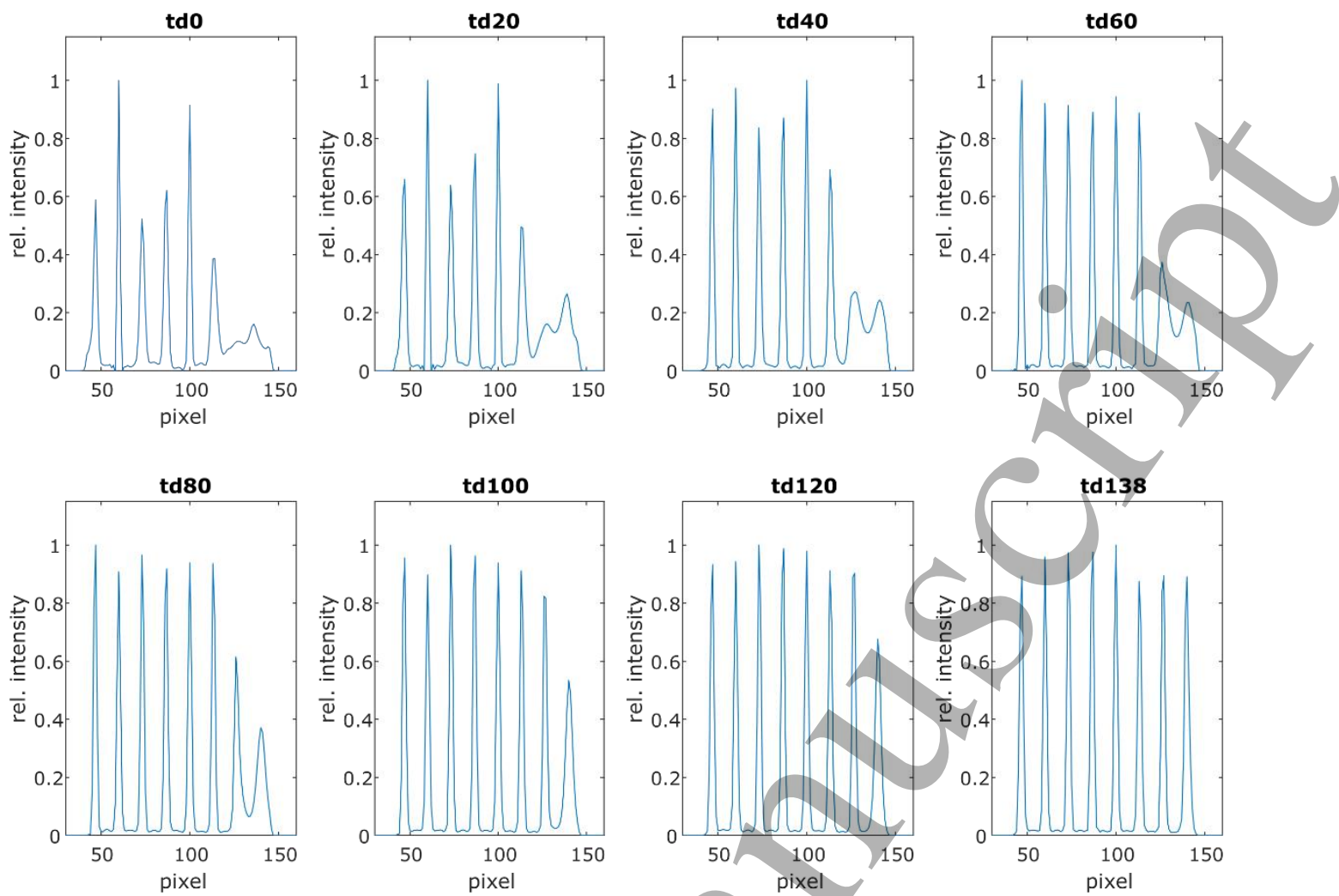

Figure 5 Axial profile of the MPH SPECT images of the custom-made multi-disk Defrise phantom acquired with variable total table displacement $t_{d}$ of the helical acquisition. The axial profiles were obtained by summing all voxel values for each transaxial slice of the SPECT images shown in Fig. 3. Each profile was scaled to a maximum value of 1 . 


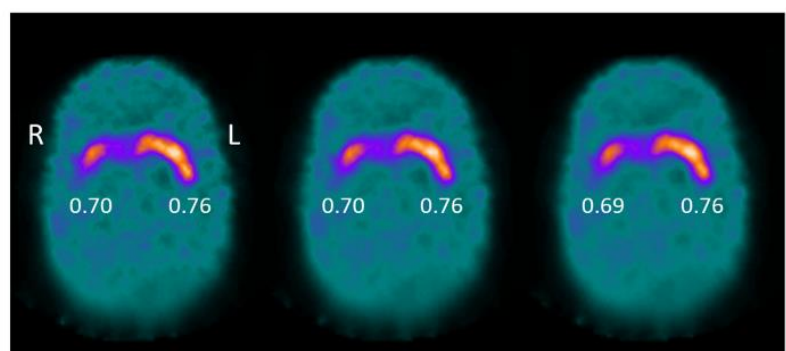

B
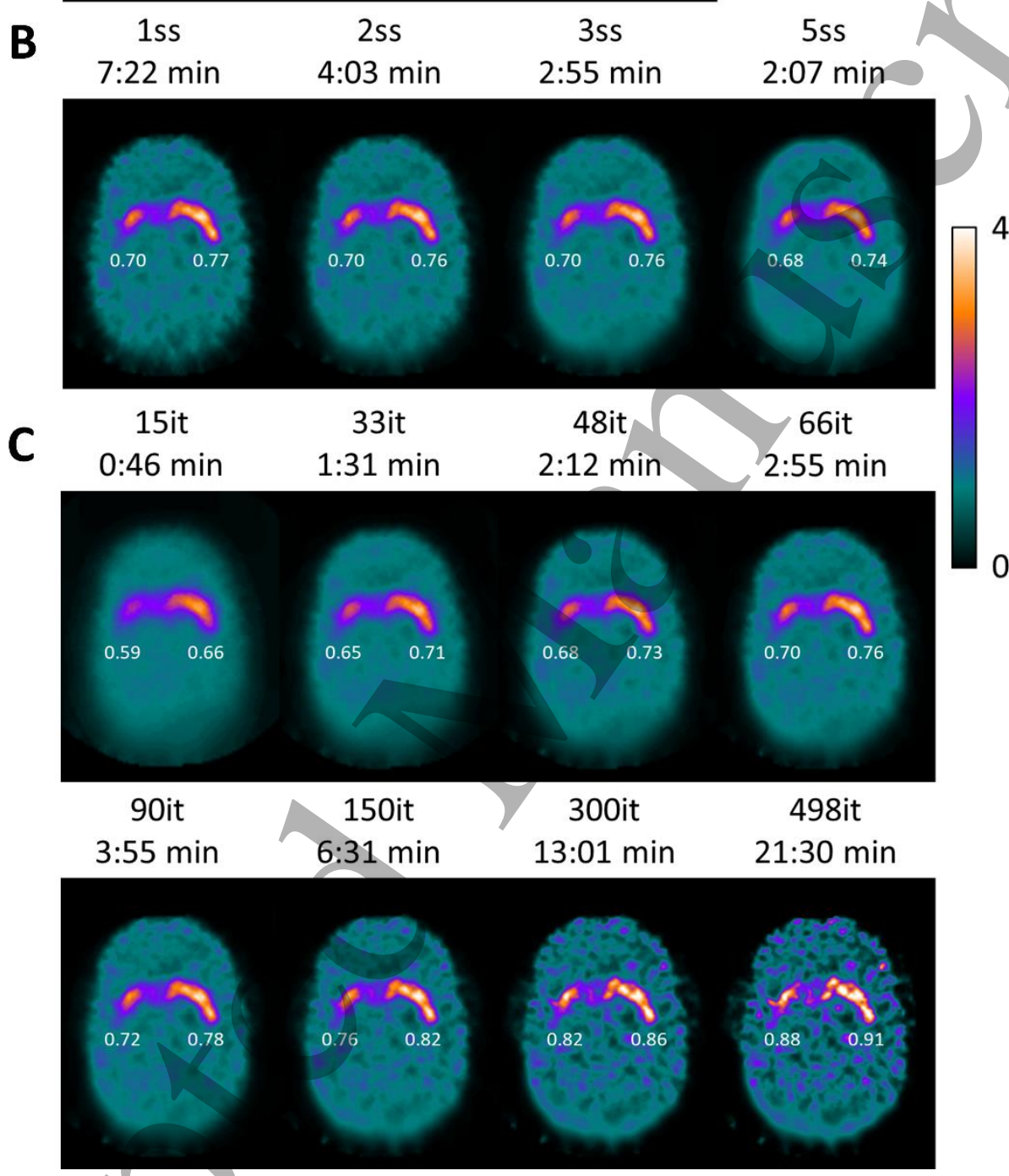

Figure 6 Central transversal slice of $2 \mathrm{~mm}$ thickness through the striatum in the MPH SPECT of the anthropomorphic striatum phantom with true 3.76:1 (left striatum) and 2.82:1 (right striatum) contrast acquired with a total number of 4.7 million counts representative of a patient scan. The images were reconstructed with varying Monte Carlo quality: low / medium / high (A, 66 effective iterations, 3 subsets), varying number of subsets (ss): 1, 2, 3, 5 (B, low Monte Carlo quality, 66 effective iterations) and varying effective number of iterations (it): 15, 33, 48, 66, 90, 150, 300, 498 (C, low Monte Carlo quality, 3 subsets). 
Post-reconstruction attenuation correction was performed using Chang's method, no scatter correction was applied. The contrast recovery coefficient for left and right putamen as well as the time required for reconstruction on the acquisition workstation $\left(\right.$ Intel $^{\circledR}$ Xeon ${ }^{\circledR}$ W-2133 CPU @ $3.60 \mathrm{GHz}, 32$ GB DDR4 RAM, Nvidia GeForce RTX 2080) of the SPECT system are specified with each slice. 


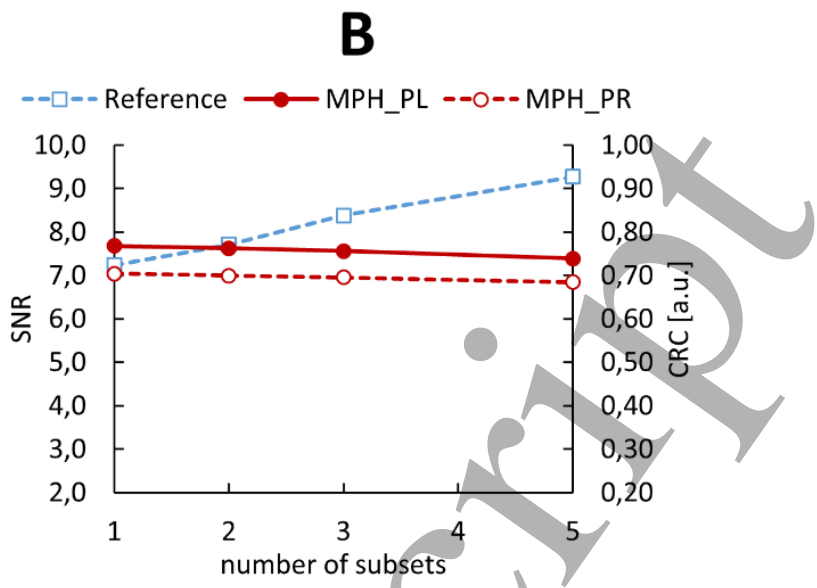

C

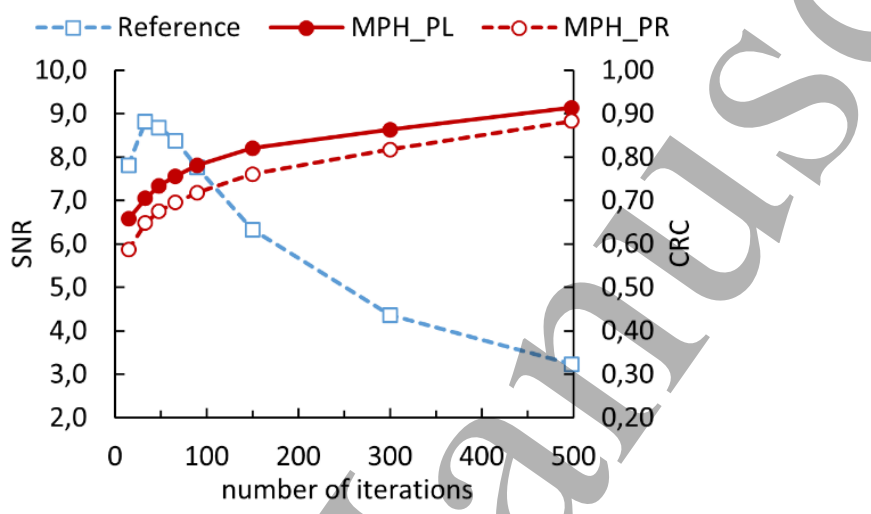

Figure 7 Quantitative analysis of the MPH SPECT images shown in figure 6. The contrast-recoverycoefficient (CRC, right y-axis) for left (PL) and right (PR) putamen (right y-axis) was computed as described in subsection "Optimization of acquisition and reconstruction protocol". The signal-to-noise (SNR, left y-axis) ratio of the voxel intensity in the reference ROI (Fig. 4B) was computed as the ratio of the mean to the standard deviation of the voxel intensity in the reference ROI. The SNR axis applies only to the blue curve, the CRC axis applies only to the red curves. CRC and SNR results are shown for varying Monte Carlo quality: low / medium / high (A, 66 effective iterations, 3 subsets), varying number of subsets: 1, 2, 3, 5 (B, low Monte Carlo quality, 90 effective iterations) and varying effective number of iterations: 15, 33, 48, 66, 90, 150, 300, 498 (C, low Monte Carlo quality, 3 subsets). 

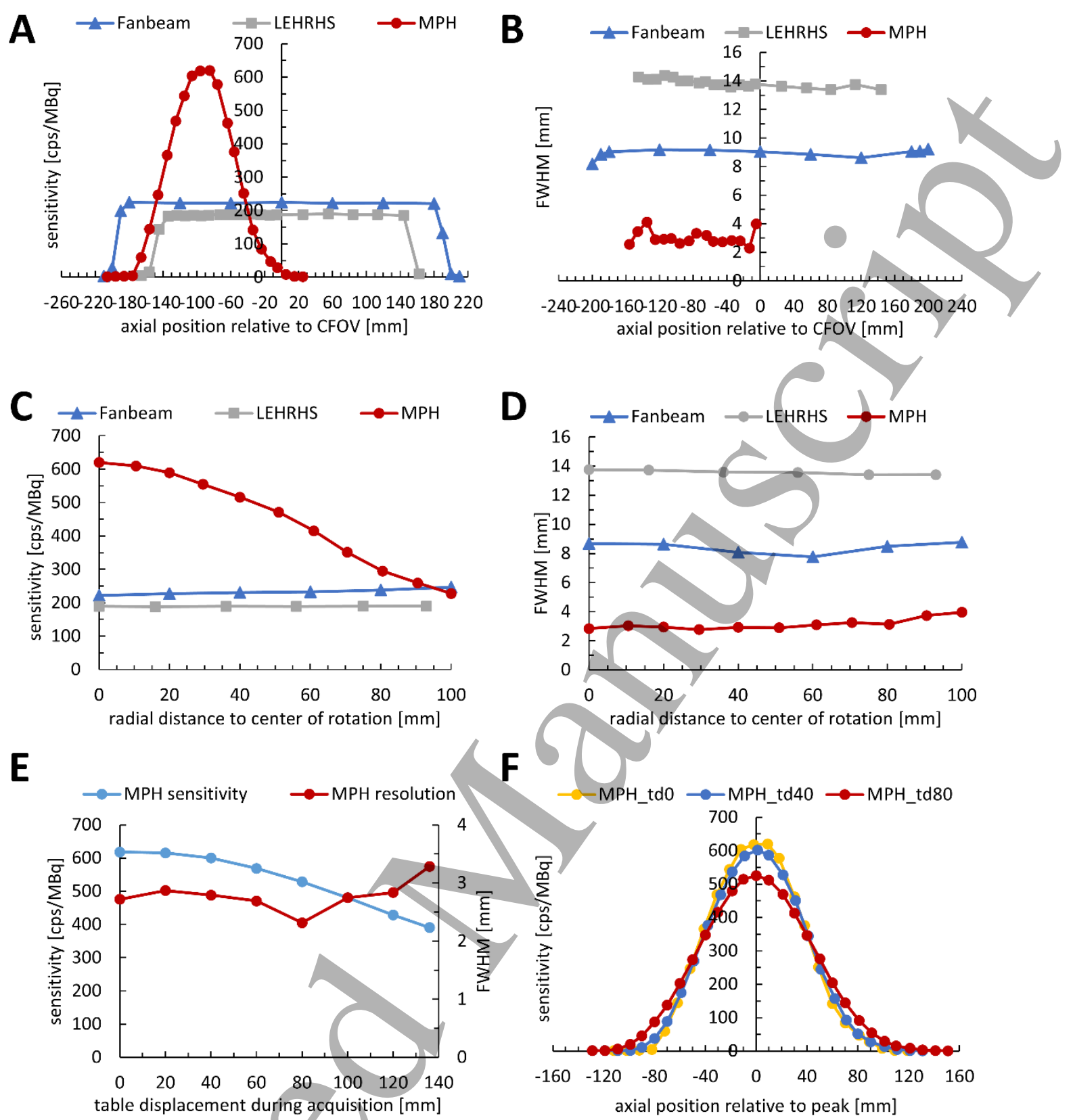

Figure 8 System count sensitivity profile of the triple-head SPECT system with the MPH collimators compared to the double-head SPECT systems with fan-beam and LEHRHS collimators in axial direction along the center of rotation axis (A) and in transaxial direction at the maximum of the axial sensitivity profile (C). Parts B and D show the FWHM of the reconstructed point source images across the FOV. MPH SPECT images were acquired and reconstructed with parameter settings optimized for DAT SPECT in clinical routine ( $\mathrm{n}_{\mathrm{v}}=90, \mathrm{t}_{\mathrm{d}}=40$, low Monte Carlo quality, 90 effective iterations, 3 subsets), fan-beam SPECT images were reconstructed with the ordered-subsets-expectation-maximization algorithm with resolution recovery implemented in the HybridRecon-Neurology tool of the Hermes SMART workstation v1.6 with parameter settings recommended for clinical DAT SPECT by Hermes. Part E shows peak system 
sensitivity (left axis) and FWHM of the reconstructed point source image (right axis) at peak location of the triple-head SPECT with MPH collimators for varying total table displacement during helical scanning. Part $\mathbf{F}$ shows complete axial profiles of system sensitivity for selected table displacements. 

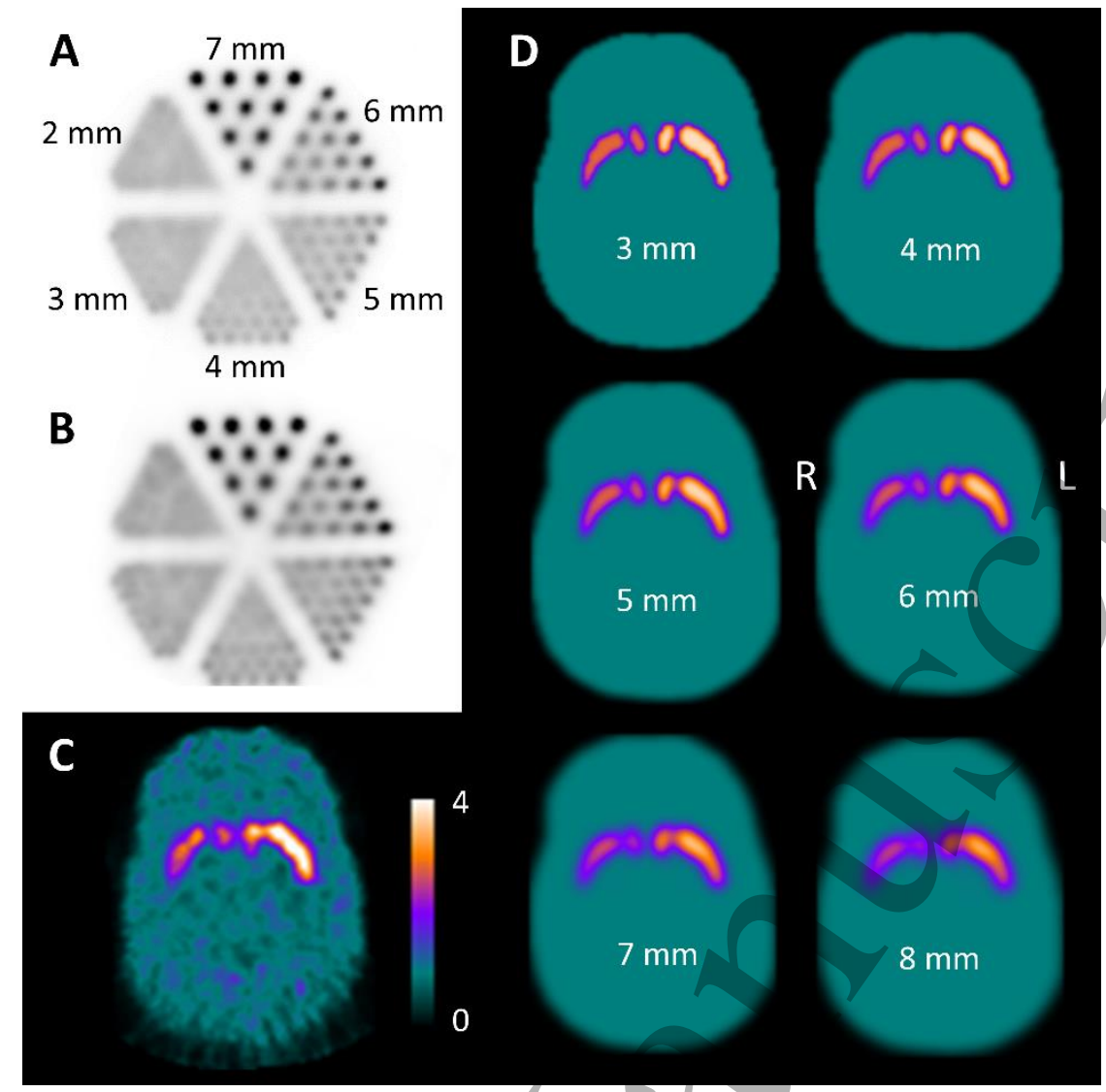

Figure 9 Transaxial images of the Derenzo type hot rod phantom filled with ${ }^{99 \mathrm{~m}} \mathrm{Tc}$ (A) or ${ }^{123} \mathrm{I}(\mathbf{B})$. Acquisition and reconstruction were performed with the parameter settings optimized for clinical DAT SPECT. The image was obtained by summing 16 transaxial slices of $1.72 \mathrm{~mm}$ thickness through the rods (resulting in of $27.5 \mathrm{~mm}$ thickness) in order to reduce statistical noise. (C): Central transversal image of 2 mm thickness through the striatum in MPH SPECT of the anthropomorphic striatum phantom with true 4:1 (left striatum) and 3:1 (right striatum) contrast acquired with a total number of 17.5 million counts. Acquisition and reconstruction were performed with the optimized settings for clinical DAT SPECT $\left(\mathrm{n}_{\mathrm{v}}=\right.$ $90, \mathrm{t}_{\mathrm{d}}=40 \mathrm{~mm}$, low Monte Carlo quality, 90 effective iterations, 3 subsets). CT-based attenuation and scatter correction was performed during iterative reconstruction. (D): Simulated SPECT images of $2 \mathrm{~mm}$ thickness of the anthropomorphic striatum phantom with 4:1 and 3:1 striatum-to-background contrast with $3,4, \ldots, 8 \mathrm{~mm}$ spatial resolution (without statistical noise). 


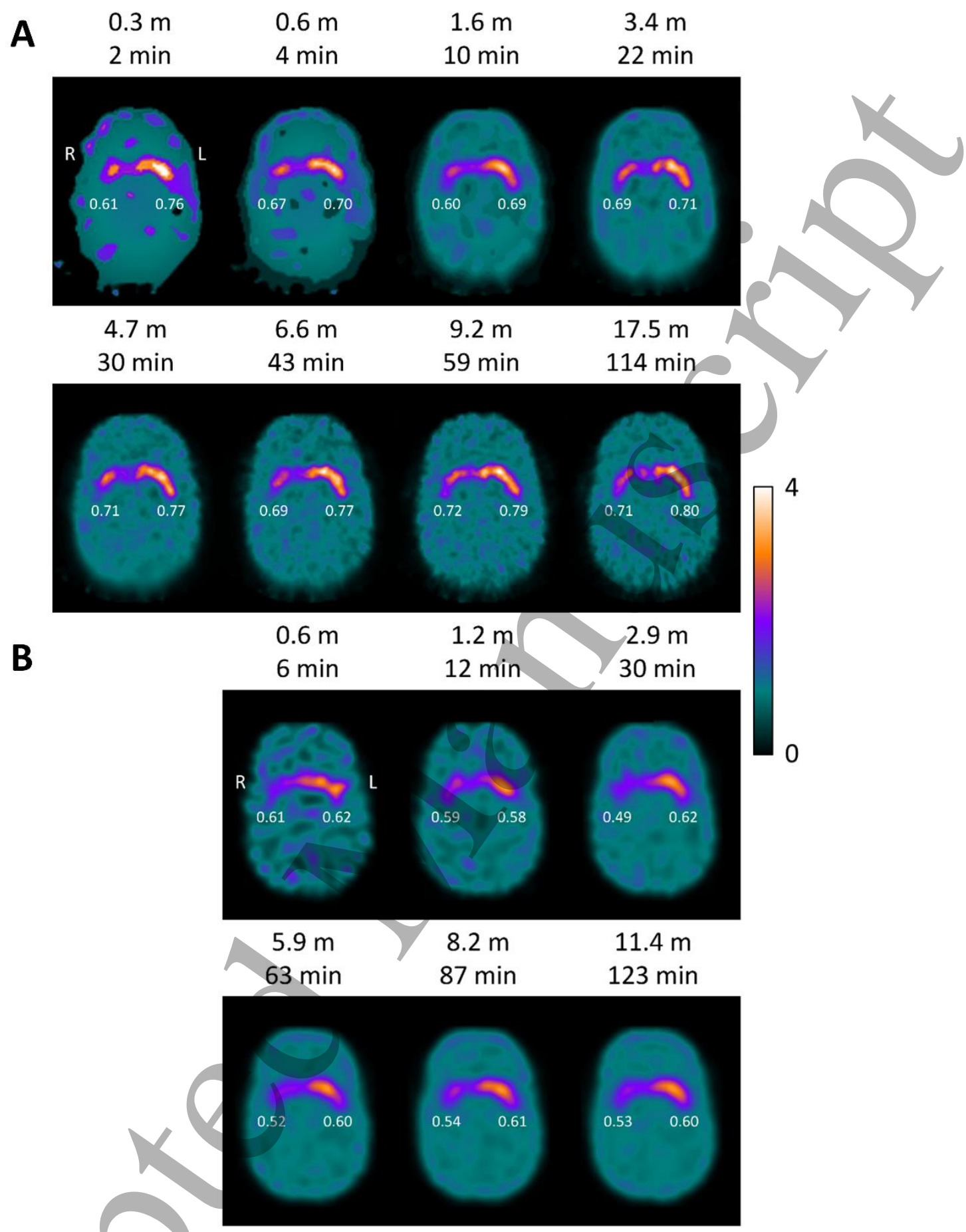

Figure 10 Central transversal slice of $2 \mathrm{~mm}$ thickness through the striatum of the anthropomorphic striatum phantom with true 4:1 (left striatum) and 3:1 (right striatum) contrast and realistic filling (background activity about $10 \mathrm{kBq} / \mathrm{ml}$ ) acquired with varying scan duration resulting in varying total number of counts $(\mathrm{m}=$ million $)$. The images were acquired with the triple-head system with MPH collimators $(\mathbf{A})$ or the double-head system with fan-beam collimators (B). MPH images were reconstructed with the parameter settings optimized for DAT SPECT in clinical routine, fan-beam SPECT images were reconstructed with 
the ordered-subsets-expectation-maximization algorithm with resolution recovery implemented in the HybridRecon-Neurology tool of the Hermes SMART workstation v1.6 with parameter settings recommended for clinical DAT SPECT by Hermes. Post-reconstruction attenuation correction using Chang's method was performed with a custom-made MATLAB script in all cases. The contrast recovery coefficient for left and right putamen is specified for each slice. 


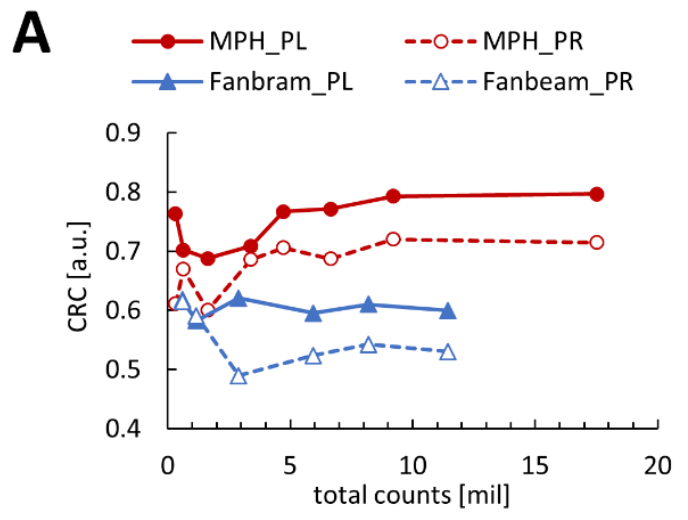

B $\cdots \cdot \cdots$ MPH_Ref. $\cdots \cdot \Delta \cdot \cdots$ Fanbeam_Ref.

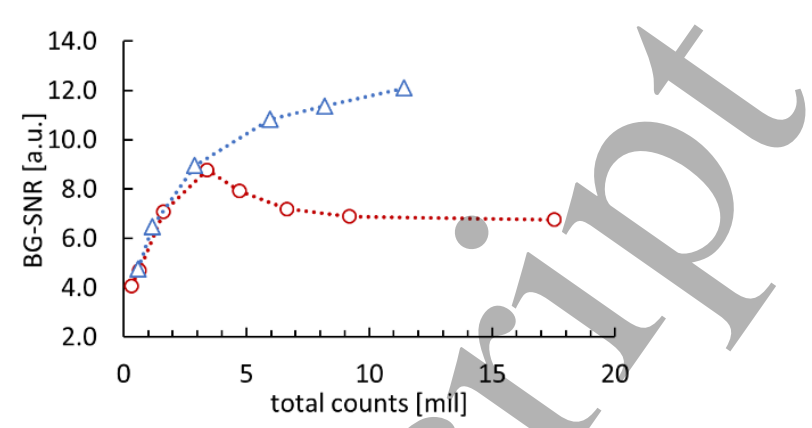

Figure 11 Quantitative analysis of the SPECT images shown in figure 10. The contrast-recoverycoefficient (CRC, A) for left (PL) and right (PR) putamen was computed as described in subsection "Optimization of acquisition and reconstruction protocol". The signal-to-noise (SNR, B) ratio of the voxel intensity in the background (BG) reference ROI (Fig. 4B) was computed as the ratio of the mean to the standard deviation of the voxel intensity in the reference ROI. CRC and BG-SNR results are given as function of the total number of acquired counts. 


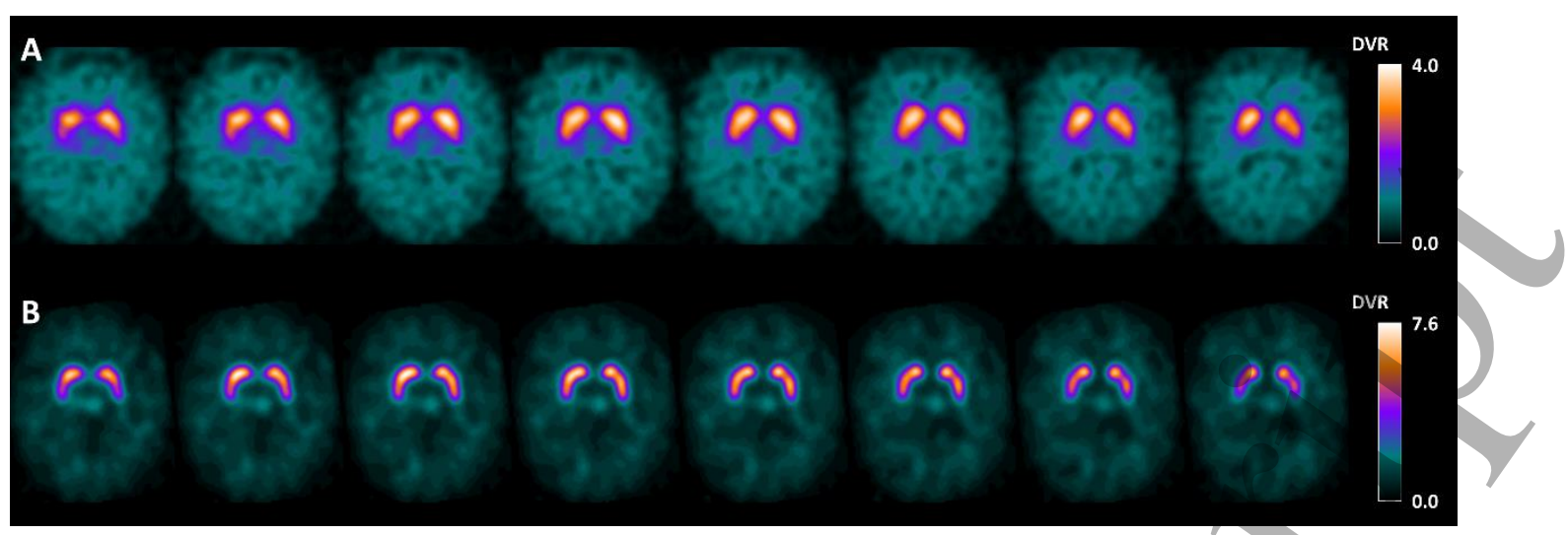

Figure 12 Normal DAT availability in a male patient (54 y) referred to DAT SPECT because of suspicion of PD. The patient was scanned twice after a single ${ }^{123} \mathrm{I}-\mathrm{FP}-\mathrm{CIT}$ injection (182 MBq). (A): 40 min scan in double-head mode with the triple-head system equipped with LEHRHS collimators that was started $3 \mathrm{~h}$ 10min after tracer injection. (B): 30 min scan with the triple-head system equipped with MPH collimators in triple-head mode that was started $5 \mathrm{~h} 7 \mathrm{~min}$ after tracer injection. Both images were reconstructed with the parameter settings optimized for DAT SPECT in clinical routine. 